\title{
Geometric Properties of Grassmannian Frames for $\mathbb{R}^{2}$ and $\mathbb{R}^{3}$
}

\author{
John J. Benedetto and Joseph D. Kolesar \\ Department of Mathematics, University of Maryland, College Park, MD 20742, USA
}

Received 16 September 2004; Revised 19 January 2005; Accepted 21 January 2005

\begin{abstract}
Grassmannian frames are frames satisfying a min-max correlation criterion. We translate a geometrically intuitive approach for two- and three-dimensional Euclidean space $\left(\mathbb{R}^{2}\right.$ and $\left.\mathbb{R}^{3}\right)$ into a new analytic method which is used to classify many Grassmannian frames in this setting. The method and associated algorithm decrease the maximum frame correlation, and hence give rise to the construction of specific examples of Grassmannian frames. Many of the results are known by other techniques, and even more generally, so that this paper can be viewed as tutorial. However, our analytic method is presented with the goal of developing it to address unresovled problems in $d$-dimensional Hilbert spaces which serve as a setting for spherical codes, erasure channel modeling, and other aspects of communications theory.
\end{abstract}

Copyright ( 2006 Hindawi Publishing Corporation. All rights reserved.

\section{INTRODUCTION}

A finite frame $\left\{x_{k}\right\}_{k=1}^{N} \subseteq \mathbb{R}^{d}, \mathbb{R}^{d}$ is $d$-dimensional Euclidean space, is characterized by the property that its span is $\mathbb{R}^{d}$, see [1]. The norm $\|x\|$ of $x \in \mathbb{R}^{d}$ is the usual Euclidean distance. Given a finite frame for $\mathbb{R}^{d}$ with $N$ elements, we would like to measure the correlation between frame elements and in particular to decide when the correlation is small. We consider the following metric which is similar to an $\ell^{\infty}$ norm [2].

Definition 1. Let $N \geq d$ and let $X_{d}^{N}=\left\{x_{k}\right\}_{k=1}^{N}$ be a subset of $\mathbb{R}^{d}$ with each $\left\|x_{k}\right\|=1$. The maximum correlation of $X_{d}^{N}$, $\mathcal{M}_{\infty}\left(X_{d}^{N}\right)$, is defined as

$$
\mathcal{M}_{\infty}\left(X_{d}^{N}\right)=\max _{k \neq l}\left|\left\langle x_{k}, x_{l}\right\rangle\right| .
$$

Note that because we consider the absolute value of the inner product rather than just the inner product, if the angle between a pair of vectors is closer to $90^{\circ}$, then the pair is less correlated, while if the angle is closer to $0^{\circ}$ or $180^{\circ}$, then the pair is more correlated. Thus, we are measuring the smaller angle between the lines (one-dimensional subspaces) spanned by these vectors. We could instead consider an $\ell^{1}-$, $\ell^{2}$-, or $\ell^{P}$-type norm to measure correlation, that is,

$$
\mathcal{M}_{p}\left(X_{d}^{N}\right)=\left(\sum_{k \neq l}\left|\left\langle x_{k}, x_{l}\right\rangle\right|^{p}\right)^{1 / p},
$$

or even weighted versions of (2). (See [3] for a discussion of the case $p=1,2$.)

Fix $d$ and $N$ with $N \geq d$. Our goal is to construct $N$-element unit-norm frames, $X_{d}^{N}$, with smallest maximum correlation, $\mathcal{M}_{\infty}\left(X_{d}^{N}\right)$, that is, unit-norm frames that are maximally spread apart. To this end, we make the following definition.

Definition 2. Let $N \geq d$. A sequence $U_{d}^{N}=\left\{u_{k}\right\}_{k=1}^{N} \subseteq \mathbb{R}^{d}$ of unit-norm vectors is an $(N, d)$-Grassmannian frame if it is a frame and if

$$
\mathcal{M}_{\infty}\left(U_{d}^{N}\right)=\inf \left\{\mathcal{M}_{\infty}\left(X_{d}^{N}\right)\right\}
$$

where the infimum is taken over all unit-norm, $N$-element frames for $\mathbb{R}^{d}$.

A compactness argument shows that Grassmannian frames exist (see Appendix A), but constructing Grassmannian frames is challenging [4-6]. As is described in [2], the concept of Grassmannian frames is related to several other areas of mathematics and engineering, for example, packings in Grassmannian spaces, spherical codes and designs, the construction of equiangular lines, strongly regular graphs, and reduction of losses associated with packet-based communications systems such as the Internet, [7-9].

In this paper, we give an analytic construction of Grassmannian frames in $\mathbb{R}^{d}$, when $d=2,3$. The first treatment of this construction problem in the case $d=3$ is found in [10]. There are extensive computational and theoretical results in [4] which approach this construction problem from a sphere packing point of view. The relevance of such constructions was brought to the attention of the frame community in [2].

After stating some technical preliminaries in Section 2, we characterize all $(N, 2)$-Grassmannian frames in Section 3. 
In Section 4, we state and prove a modest generalization of a theorem, given in [2], which provides a lower bound for $\mathcal{M}_{\infty}\left(X_{d}^{N}\right)$. Section 5 is devoted to the construction of $(4,3)$ Grassmannian frames from first principles rather than using the theorem in Section 4. Convexity arguments are used in Section 6 to construct examples of $(5,3)$-Grassmannian frames. In Section 7, we construct a $(6,3)$-Grassmannian frame using the theorem in Section 4. The techniques used in the constructions of Sections 5, 6, and 7 were developed in part to fathom the geometrical ideas of Fejes Tóth [10].

Our presentation is technical and, we believe, necessarily so. Some of the technicalities are routine, but they are included since going from one step to the next without exhibiting them sometimes seemed mysterious. On the other hand, some of our techniques may very well be useful in developing more general methods of finding frames with small correlations in applicable complex and higher-dimensional settings. For example, see the new techniques used in proving Propositions 2 and 3 and Lemmas 4 and 5. Our notation is standard, but we do mention that " $\Longrightarrow$ " means "implies" and " $\Longleftrightarrow$ " means "if and only if".

\section{PRELIMINARIES}

In this section, we collect some definitions and theorems used in the sequel.

The transpose of a vector or matrix $A$ is denoted by $A^{T}$; and the Hermitian transpose of a vector or matrix $B$ with complex entries is denoted by $B^{*}$, the conjugate transpose of $B$, that is, $B^{*}=\bar{B}^{T}$. A $d \times d$ matrix $U$ with real entries is orthogonal if the columns of $U$ are orthonormal, that is, $U^{T} U=I_{d}$, where $U^{T}$ is the transpose of $U$ and $I_{d}$ is the $d \times d$ identity matrix. If $U$ is orthogonal, that is, $U \in S O_{d}$, then for any $x, y \in \mathbb{R}^{d},\|U x\|=\|x\|$ and $\langle U x, U y\rangle=\langle x, y\rangle$.

The torus is $\mathbb{T}_{2 \pi}=\mathbb{R} /(2 \pi \mathbb{Z})$. We take any fixed half-open interval of length $2 \pi$ to be a representative of $\mathbb{T}_{2 \pi}$.

The unit sphere in $\mathbb{R}^{d}$ is $S^{d-1}=\left\{x \in \mathbb{R}^{d}:\|x\|=1\right\}$. A set $\left\{x_{1}, \ldots, x_{N}\right\}$ of unit-norm vectors is equiangular if there is an $\alpha \in[0,1]$ such that $\left|\left\langle x_{k}, x_{l}\right\rangle\right|=\alpha$ when $k \neq l$.

A $d \times d$ matrix $A$ with real entries is symmetric if $A^{T}=A$.

The spectral theorem for symmetric matrices is the following, see [11]. We use it in Theorem 3.

Theorem 1 (spectral theorem). A $d \times d$ symmetric matrix $A$ over $\mathbb{R}$ has the following properties.

(i) A has d real eigenvalues counting multiplicities.

(ii) The dimension of the eigenspace for each eigenvalue $\lambda$ equals the multiplicity of $\lambda$ as a root of the characteristic equation $\operatorname{det}(A-\lambda I)=0$.

(iii) The eigenspaces are mutually orthogonal in the sense that eigenvectors corresponding to different eigenvalues are orthogonal.

(iv) $A$ is orthogonally diagonalizable, that is, there is an orthonormal basis of eigenvectors for A.

We now state some basic definitions of frame theory $[1,3$, 12-14]. Let $\mathscr{H}$ be a separable Hilbert space, and let $X=\left\{x_{n}\right.$ : $n \in \ell\} \subset \mathscr{H}$, where $\ell$ is a countable indexing set. Consider the following map associated with the set $X$ :

$$
\begin{gathered}
L: \mathscr{H} \longrightarrow \ell^{2}(\ell), \\
y \longmapsto\left\{\left\langle y, x_{n}\right\rangle\right\}_{n \in \ell} .
\end{gathered}
$$

If $L$ is a well-defined linear map, that is, if $\sum_{n \in \ell}\left|\left\langle y, x_{n}\right\rangle\right|^{2}<$ $\infty$ for any $y \in \mathscr{H}$, then $L$ is a Bessel map and $X$ is a Bessel sequence. The adjoint of $L$ is the map

$$
\begin{gathered}
L^{*}: \ell^{2}(\ell) \longrightarrow \mathcal{H}, \\
\{c[n]\}_{n \in \ell} \longmapsto \sum_{n \in \ell} c[n] x_{n} .
\end{gathered}
$$

If $L$ is a Bessel map, the corresponding frame operator is the map $S: \mathcal{H} \rightarrow \mathcal{H}$ defined as $L^{*} L$. Thus, for any $y \in \mathscr{H}$,

$$
S(y)=L^{*}(L(y))=\sum_{n \in \ell}\left\langle y, x_{n}\right\rangle x_{n} .
$$

As such $y=\sum_{n \in \ell}\left\langle y, x_{n}\right\rangle S^{-1} x_{n}$. The Grammian operator is the map $G: \ell^{2}(\ell) \rightarrow \ell^{2}(\ell)$ defined as $G=L L^{*}$. Both $S$ and $G$ are positive and hence selfadjoint operators.

A Bessel sequence $X$ is a frame for $\mathscr{H}$ if there exist constants $A, B$ with $0<A \leq B<\infty$ such that, for any $y \in \mathcal{H}$,

$$
A\|y\|^{2} \leq \sum_{n \in \ell}\left|\left\langle y, x_{n}\right\rangle\right|^{2} \leq B\|y\|^{2}
$$

Thus, given any frame, we have four natural maps: $L, L^{*}, S$, and $G$. If the indexing set $\ell$ is finite, then $X$ is called a finite frame. Also, if $A=B$, then $X$ is called a tight frame or, if we wish to emphasize the bound, an A-tight frame.

Throughout this paper, we will use that fact that any finite set of vectors forms a frame for its span with the frame bounds being the largest and smallest eigenvalues of the frame operator. Since any finite set of vectors automatically has an upper frame bound by Cauchy-Schwarz, the fact that any finite set is a frame for its span is a consequence of the following result.

Proposition 1. The following three statements are equivalent:

(i) $\left\{x_{n}\right\}_{n=1}^{N}$ is a frame for $\mathbb{R}^{d}$;

(ii) $\operatorname{span}\left\{x_{n}\right\}_{n=1}^{N}=\mathbb{R}^{d}$;

(iii) there exists $A>0$ such that for all $y \in \mathbb{R}^{d}$,

$$
A\|y\|^{2} \leq \sum_{n=1}^{N}\left|\left\langle y, x_{n}\right\rangle\right|^{2} .
$$

\section{TWO-DIMENSIONAL GRASSMANNIAN FRAMES}

We classify all $(N, 2)$-Grassmannian frames. The idea for the following proof is illustrated in Figure 1. In fact, in order to maximize the minimum angle between pairs of vectors, the vectors must be equally spaced.

Theorem $2\left((N, 2)\right.$-Grassmannian). Let $X=X_{2}^{N}=\left\{x_{k}\right\}_{k=1}^{N}$ be a collection of $N$ unit vectors in $\mathbb{R}^{2}$. Then the following lower bound holds:

$$
\cos \left(\frac{\pi}{N}\right) \leq \mathcal{M}_{\infty}(X) .
$$




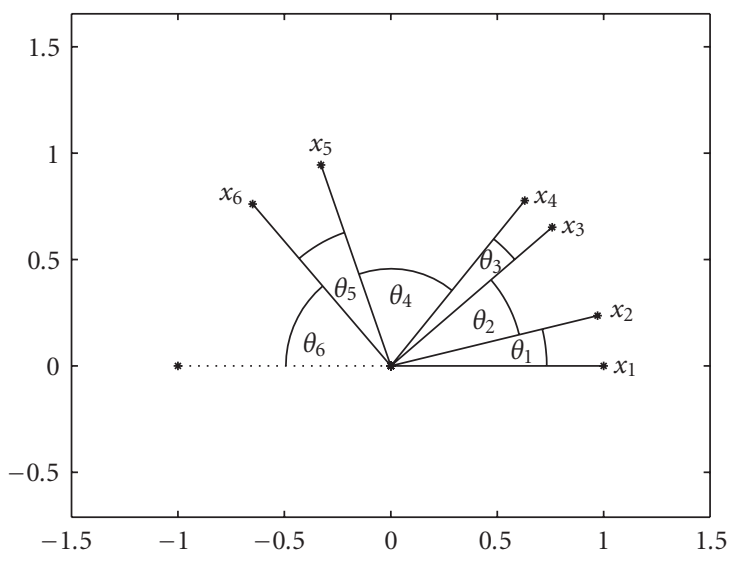

FIgURE 1: An example of the reordering induced by the inequalities on the inner products in (11) for $N=6$.

Furthermore, $X$ is an $(N, 2)$-Grassmannian frame if and only if there are $P \in S \mathrm{~S}_{2}$ and a sequence $\left\{\varepsilon_{k}\right\}_{k=1}^{N} \subset\{ \pm 1\}^{N}$ such that

$$
\begin{aligned}
P(\varepsilon X): & =\left\{P\left(\varepsilon_{k} x_{k}\right): x_{k} \in X_{2}^{N}\right\} \\
& =\left\{\left(\begin{array}{l}
\cos \left(\frac{\pi k}{N}\right) \\
\sin \left(\frac{\pi k}{N}\right)
\end{array}\right): k=1, \ldots, N\right\} .
\end{aligned}
$$

Proof. Let $\delta_{1}=(1,0)^{T}$ and let $\delta_{2}=(0,1)^{T}$. Since $|\langle x, y\rangle|=$ $|\langle x,-y\rangle|$, we note that changing the sign of any $x_{k} \in X$ does not effect the value of $\mathcal{M}_{\infty}(X)$. Thus, by changing the sign on $x_{k}$ when necessary, we may assume $x_{k} \in\left\{v \in S^{1}:\left\langle v, \delta_{2}\right\rangle \geq\right.$ $0\}$. Also, since rotations preserve inner products, applying a rotation to all the vectors in $X$ does not effect $\mathcal{M}_{\infty}(X)$. Thus, rotating by $-\phi$, where $\phi=\min _{k=1, \ldots, N} \cos ^{-1}\left(\left\langle x_{k}, \delta_{1}\right\rangle\right)$, and reordering if necessary, we may assume $x_{1}=\delta_{1}=(1,0)^{T}$, and

$$
1 \geq\left\langle x_{2}, x_{1}\right\rangle \geq\left\langle x_{3}, x_{1}\right\rangle \geq \cdots \geq\left\langle x_{N}, x_{1}\right\rangle \geq-1 .
$$

For $k=1, \ldots, N-1$, let $\theta_{k}$ be the angle between $x_{k}$ and $x_{k+1}$, and let $\theta_{N}$ be the angle between $x_{N}$ and the negative $x$-axis, that is, $\theta_{k}=\cos ^{-1}\left(\left\langle x_{k+1}, x_{k}\right\rangle\right)$ and $\theta_{N}=$ $\cos ^{-1}\left(\left\langle-\delta_{1}, x_{N}\right\rangle\right)$, see Figure 1 for an example when $N=6$. Then, because of the above reordering, $\theta_{k} \geq 0$ for $k=$ $1, \ldots, N$, and $\sum_{k=1}^{N} \theta_{k}=\pi$. Thus, for $1 \leq l<k \leq N$,

$$
\left|\left\langle x_{k}, x_{l}\right\rangle\right|=\left|\cos \left(\sum_{j=l}^{k-1} \theta_{j}\right)\right|,
$$

where

$$
\min _{k=1, \ldots, N-1} \theta_{k} \leq \sum_{j=l}^{k-1} \theta_{j} \leq \pi-\theta_{N} .
$$

Furthermore, $|\cos (\theta)|$ has a maximum on $[0, \pi]$ at $\theta=0$ and $\theta=\pi$, and $|\cos (\theta)|$ is monotone decreasing on $[0, \pi / 2]$ and monotone increasing on $[\pi / 2, \pi]$. Hence,

$$
\begin{aligned}
\mathcal{M}_{\infty}(X) & \\
& =\max _{k \neq l}\left|\cos \left(\sum_{j=l}^{k-1} \theta_{j}\right)\right| \\
& =\max \left\{\left|\cos \left(\pi-\theta_{N}\right)\right|,\left|\cos \left(\min _{k=1, \ldots, N-1} \theta_{k}\right)\right|\right\} \\
& =\left|\cos \left(\min _{k=1, \ldots, N} \theta_{k}\right)\right| .
\end{aligned}
$$

Therefore, in order to minimize $\mathcal{M}_{\infty}(X)$, we must choose $N$ positive numbers $\alpha_{1}, \ldots, \alpha_{N}$ which sum to $\pi$ and which minimize $\left|\cos \left(\min _{k=1, \ldots, N} \alpha_{k}\right)\right|$, and hence which maximize the expression

$$
\min _{k=1, \ldots, N} \alpha_{k}
$$

Now we claim that if $\alpha_{1}, \ldots, \alpha_{N}$ maximize (15), then $\alpha_{1}=$ $\cdots=\alpha_{N}$. We prove this implication by contraposition, that is, assume it is not the case that $\alpha_{1}=\cdots=\alpha_{N}$. Then there is an $m \in\{1,2, \ldots, N-1\}$ so that if we list $\alpha_{1} \leq \cdots \leq \alpha_{N}$ by size, then only the first $m$ is equal, and the $(m+1)$ th is strictly larger than the $m$ th, that is,

$$
\alpha_{k_{1}}=\alpha_{k_{2}}=\cdots=\alpha_{k_{m}}<\alpha_{k_{m+1}} \leq \cdots \leq \alpha_{k_{N}} .
$$

Let $\nu=\alpha_{k_{m+1}}-\alpha_{k_{m}}$ and, for $j=1, \ldots, N$, define the sequence $\beta_{k_{j}}$ as

$$
\beta_{k_{j}}= \begin{cases}\alpha_{k_{j}}+\frac{\nu}{2 m} & \text { for } j=1, \ldots, m, \\ \alpha_{k_{j}}-\frac{\nu}{2} & \text { for } j=m+1, \\ \alpha_{k_{j}} & \text { for } j=m+2, \ldots, N\end{cases}
$$

Now the new set,

$$
\beta_{k_{1}}=\beta_{k_{2}}=\cdots=\beta_{k_{m}} \leq \beta_{k_{m+1}} \leq \cdots \leq \beta_{k_{N}},
$$

has a strictly larger minimum angle than the original since for $j=1, \ldots, N$,

$$
\min _{k=1, \ldots, N} \alpha_{k}=\alpha_{k_{1}}<\alpha_{k_{1}}+\frac{\nu}{2 m}=\beta_{k_{1}} \leq \beta_{j} .
$$

We see that the original $\alpha s$ do not maximize (15). So by contraposition we have that if the $\alpha s$ maximize (15), then they must all be equal. Finally, if $\alpha$ is the common value, then $\sum_{k=1}^{N} \alpha_{k}=N \alpha=\pi$, and therefore $\alpha=\pi / N$. Thus, $\pi / N \geq \min _{k=1, \ldots, N} \theta_{K}$, and therefore

$$
\cos \left(\frac{\pi}{N}\right) \leq \cos \left(\min _{k=1, \ldots, N} \theta_{K}\right)=\mathcal{M}_{\infty}(X) .
$$

Next we prove that any $(N, 2)$-Grassmannian frame is, up to a sign change, the first $N$ adjacent vertices of a regular $2 N$ gon. If $X$ is an $(N, 2)$-Grassmannian frame, then, using the above argument, we see that we can choose $\left\{\varepsilon_{k}\right\} \subset\{ \pm 1\}^{N}$ and $\mathrm{P} \in \mathrm{SO}_{2}$ so that the frame

$$
P(\varepsilon X)=\left\{P\left(\varepsilon_{k} x_{k}\right): x_{k} \in X\right\}
$$


is in the closed upper half-plane with one of the vectors being $(1,0)^{T}$; and

$$
\mathcal{M}_{\infty}(X)=\mathcal{M}_{\infty}(P(\varepsilon X))=\cos \left(\min _{k=1, \ldots, N} \theta_{k}\right),
$$

where $\theta_{k}$ is the angle between the $k$ th and $(k+1)$ th adjacent vectors in $P(\varepsilon X)$ (reindexing may be necessary). Since an $(N, 2)$-Grassmannian frame minimizes the $\infty$-correlation $\mathcal{M}_{\infty}(X)$, the above argument also shows that $\theta_{1}=\cdots=$ $\theta_{N}=\pi / N$. Therefore, the angle between adjacent vectors in $P(\varepsilon X)$ is $\pi / N$, and we have proved the forward direction of the equivalence.

To show the reverse implication, we note that if

$$
P(\varepsilon X)=\left\{\left(\begin{array}{c}
\cos \left(\frac{\pi k}{N}\right) \\
\sin \left(\frac{\pi k}{N}\right)
\end{array}\right): k=1, \ldots, N\right\},
$$

then

$$
\begin{aligned}
\mathcal{M}_{\infty}(X) & =\mathcal{M}_{\infty}(P(\varepsilon X)) \\
& =\cos \left(\min _{k=1, \ldots, N} \theta_{k}\right)=\cos \left(\frac{\pi}{N}\right) .
\end{aligned}
$$

Hence, $X$ is $(N, 2)$-Grassmannian since it achieves the lower bound.

Notice that for $N$ odd, if we change the sign on the $N$ th roots of unity below the real axis, then we obtain the frame described in the above claim with $\varepsilon_{k}=1$, that is, with all vectors in the upper half-plane, and a common angle of $\pi / \mathrm{N}$ between adjacent vectors. Hence, for $N$ odd, the $N$ th roots of unity are $(N, 2)$-Grassmannian. Furthermore, for $N$ even, the $N$ th roots of unity do not form an $(N, 2)$-Grassmannian frame because $\zeta$ and $-\zeta$ are both $N$ th roots. If we identify $\zeta$ and $-\zeta$, then we obtain an $(N / 2,2)$-Grassmannian frame.

\section{A LOWER BOUND FOR $\mathcal{M}_{\infty}$}

It is more difficult to construct a Grassmannian frame in $\mathbb{R}^{3}$ for $N>3$ than in $\mathbb{R}^{2}$. Thus, we first derive a lower bound for the maximum correlation between frame elements of an $N$-element frame for $\mathbb{R}^{d}$, see $[2,15]$ for superb treatments, although we have felt compelled to spell out all details. Such lower bounds are useful in coding theory [16], and we first learned of them in [2].

The proofs of the following lemma and theorem are found in Appendix B.

Lemma 1. Let $H_{n}$ be the $n \times n$ matrix with 1 on the main diagonal and $\beta$ elsewhere, and let $C_{n}$ be the $n \times n$ matrix defined by

$$
\left[C_{n}\right]_{i, j}= \begin{cases}\beta, & \text { if }(i, j)=(1,1), \\ {\left[H_{n}\right]_{i, j},} & \text { otherwise }\end{cases}
$$

where $\left[H_{n}\right]_{i, j}$ is the $(i, j)$ th entry of the matrix $H_{n}$. Then

$$
\begin{gathered}
\operatorname{det}\left(H_{n}\right)=(1+(n-1) \beta)(1-\beta)^{n-1}, \\
\operatorname{det}\left(C_{n}\right)=\beta(1-\beta)^{n-1} .
\end{gathered}
$$

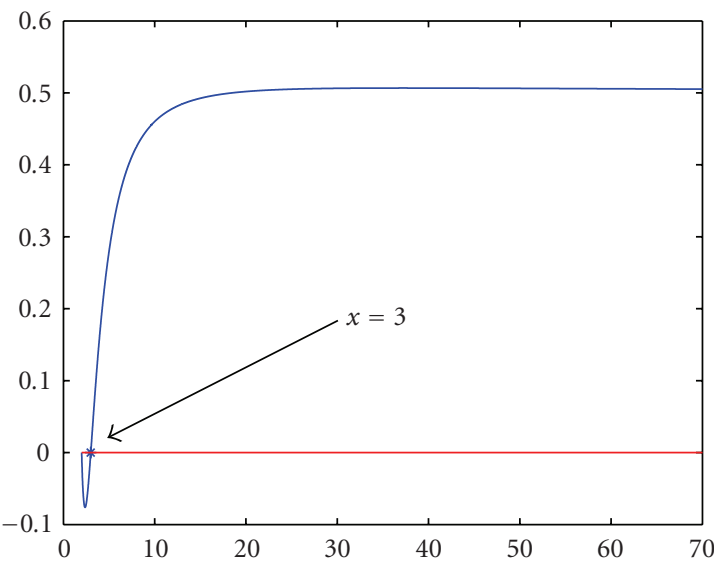

Figure 2: The function $f(x)=\cos ^{2}(\pi / x)-(x-2) /(2(x-1))$ on $[2,70]$.

Theorem 3. Let $N \geq d$, let $X_{d}^{N}$ be an $N$-element subset of $S^{d-1}$, and let $d_{0}=\operatorname{dim}\left(\operatorname{span}\left(X_{d}^{N}\right)\right)$. Then

$$
\mathcal{M}_{\infty}\left(X_{d}^{N}\right) \geq \sqrt{\frac{N-d_{0}}{d_{0}(N-1)}},
$$

where equality holds in (27) if and only if

(a) $X_{d}^{N}$ is equiangular,

(b) $X_{d}^{N}$ is a tight frame for its span with frame bounds $A=B=N / d_{0}$.

Furthermore, if $N>(d(d+1)) / 2$, then $X_{d}^{N}$ is not equiangular, hence equality cannot hold in (27).

Remark. Theorem 2 shows that $\mathcal{M}_{\infty}\left(X_{2}^{N}\right) \geq \cos (\pi / N)$, while Theorem 3 shows $\mathcal{M}_{\infty}\left(X_{2}^{N}\right) \geq \sqrt{(N-2) /(2(N-1))}$. Using standard calculus techniques, we can show that the equality in Theorem 2 is an improvement over the bound in Theorem 3 for all $N>3$. Let

$$
f(x)=\cos ^{2}\left(\frac{\pi}{x}\right)-\frac{x-2}{2(x-1)}
$$

(see Figure 2), so that

$$
\begin{gathered}
f^{\prime}(x)=\frac{\pi}{x^{2}} \sin \left(\frac{2 \pi}{x}\right)-\frac{1}{2(x-1)^{2}}, \\
f^{\prime \prime}(x)=-\frac{2 \pi}{x^{3}}\left(\frac{\pi}{x} \cos \left(\frac{2 \pi}{x}\right)+\sin \left(\frac{2 \pi}{x}\right)\right)+\frac{1}{(x-1)^{3}} .
\end{gathered}
$$

Hence,

$$
f^{\prime}(x)>0 \Leftrightarrow \sin \left(\frac{2 \pi}{x}\right)>\frac{1}{2 \pi}\left(\frac{x}{x-1}\right)^{2} .
$$

For $x \in[3,6]$,

$$
\sin \left(\frac{2 \pi}{x}\right) \geq \frac{\sqrt{3}}{2} \geq \frac{9}{8 \pi} \geq \frac{1}{2 \pi}\left(\frac{x}{x-1}\right)^{2},
$$

and so $f(x)$ is increasing for $x \in[3,6]$, and since $f(3)=$ 0 , we have that $f(x) \geq 0$ for $x \in[3,6]$. Furthermore, for 
TABLE 1: Improvement of the optimal bound derived in Theorem 3 for the case of $(N, 2)$-Grassmannian frames.

\begin{tabular}{lcc}
\hline$N$ & $\begin{array}{c}\text { Optimal bound } \\
=\sqrt{\frac{N-2}{2(N-1)}}\end{array}$ & $\begin{array}{c}\text { Bound from Theorem 2 } \\
\cos \left(\frac{\pi}{N}\right)\end{array}$ \\
\hline 3 & 0.5000 & 0.5000 \\
4 & 0.5774 & 0.7071 \\
5 & 0.6124 & 0.8090 \\
6 & 0.6325 & 0.8660 \\
7 & 0.6455 & 0.9010 \\
8 & 0.6547 & 0.9239 \\
9 & 0.6614 & 0.9397 \\
10 & 0.6667 & 0.9511 \\
\hline
\end{tabular}

$x \in[6, \infty), 36 / 50 \pi \geq(1 / 2 \pi)(x /(x-1))^{2}$. Also, $\sin (2 \pi / x) \geq$ $36 / 50 \pi$ if and only if

$$
x \leq \frac{2 \pi}{\sin ^{-1}(36 / 50 \pi)}<27.172 .
$$

Thus, $f(x)$ is increasing for $x \in[3,27]$ and hence it is greater than zero on that same interval. We also note that for $x>1$,

$$
\frac{1}{2 \pi}<\frac{1}{2 \pi}\left(\frac{x}{x-1}\right)^{2} .
$$

Further, $\sin (2 \pi / x) \leq 1 / 2 \pi$ when

$$
x \geq \frac{2 \pi}{\sin ^{-1}(1 / 2 \pi)}>39.310 .
$$

Hence, $f$ is decreasing on the interval $[40, \infty)$, and since $\lim _{x \rightarrow \infty} f(x)=1 / 2$, we have that $f(x)>1 / 2$ for $x \in[40, \infty)$. Finally, we check that $f^{\prime \prime}<0$ on the interval $[27,40]$ and $f(27), f(40)>1 / 2$; thus, $f(x)>1 / 2$ on $[27,40]$. In summary we have shown that $f(x)>0$ on $(3, \infty)$ and that $f(x)>1 / 2$ on $[27, \infty)$. Therefore,

$$
\cos \left(\frac{\pi}{N}\right)>\sqrt{\frac{N-2}{2(N-1)}} \text { for } N>3
$$

and we see that Theorem 2 is an improvement over Theorem 3 in the case $d=2$, see Table 1 .

In light of Theorem 3, we make the following definition.

Definition 3. Let $N, d \in \mathbb{N}$ with $d \leq N \leq d(d+1) / 2$. Let $X_{d}^{N}=\left\{x_{k}\right\}_{k=1}^{N}$ be a frame for $\mathbb{R}^{d}$ with $\left\|x_{k}\right\|=1$. We call $X_{d}^{N}$ an optimal Grassmannian frame if $X_{d}^{N}$ satisfies (27) with equality, that is,

$$
\mathcal{M}_{\infty}\left(X_{d}^{N}\right)=\sqrt{\frac{N-d}{d(N-1)}} .
$$

In $\mathbb{R}^{2}$, since $d=2$ and $d(d+1) / 2=3$, only frames with $N=2$ and $N=3$ elements can be optimal Grassmannian.
TABLE 2: Bounds for $N$-element frames in $\mathbb{R}^{3}$ with potential of being optimal Grassmannian.

\begin{tabular}{ccc}
\hline$N$ & $\begin{array}{c}\text { Optimal bound } \\
=\sqrt{\frac{N-3}{3(N-1)}}\end{array}$ & $\begin{array}{c}\text { Grassmannian bound } \\
=\min \mathcal{M}_{\infty}\left(X_{3}^{N}\right)\end{array}$ \\
\hline 3 & 0 & 0 \\
4 & $0.333 \overline{3}$ & $0.333 \overline{3}$ \\
5 & 0.4082 & 0.4472 \\
6 & 0.4472 & 0.4472 \\
\hline
\end{tabular}

Since $\cos (\pi / 2)=0=\sqrt{(2-2) / 2(2-1)}$, and $\cos (\pi / 3)=$ $1 / 2=\sqrt{(3-2) / 2(3-1)}$, both $(2,2)$ - and $(3,2)$-Grassmannian frames are optimal. The same phenomenon does not happen in three dimensions. Table 2 lists the Grassmannian bound which will be proven below and the optimal bound for $N=3,4,5,6$, (the only $N s$ with the possibility of being optimal). By inspecting Table 2 , we notice that $(5,3)$ Grassmannian frames are not optimal, while $(3,3)-,(4,3)$ and $(6,3)$-Grassmannians are optimal.

\section{5. (4,3)-GRASSMANNIAN FRAMES}

In this section and the next two, we will derive the bounds for three-dimensional Grassmannian frames with $N=3,4,5$, and 6. First note that if $N=3$, and if $X$ is any orthonormal basis for $\mathbb{R}^{3}$, then $0 \leq \mathcal{M}_{\infty}(X)=0$. Hence, any orthonormal basis is Grassmannian and, in fact, $X$ is trivially optimal Grassmannian.

Next consider $N=4$. We need the following two lemmas which are necessary to rigorize Fejes Tóth's ideas in [10]. In particular, Lemma 2 is intuitively elementary when we consider the fact that the $\ell^{2}$-norm is convex, $Q$ is convex, and $C$ is the set of extreme points of $Q$.

Lemma 2. Let $a \in \mathbb{R}^{d}$ and let $\left\{v_{1}, v_{2}, \ldots, v_{d}\right\} \subset \mathbb{R}^{d}$. Set

$$
\begin{aligned}
& Q=\left\{a+\sum_{j=1}^{d} s_{j} v_{j}: s_{j} \in[0,1]\right\}, \\
& C=\left\{a+\sum_{j=1}^{d} \varepsilon_{j} v_{j}: \varepsilon_{j} \in\{0,1\}\right\},
\end{aligned}
$$

and choose $c \in C$ such that $\|c\|=\max \left\{\left\|c_{l}\right\|: c_{l} \in C\right\}$, where $l=1, \ldots, 2^{d}$. Then, for any $v \in Q \backslash C,\|v\|<\|c\|$.

Lemma 2 is used in the proof of Lemma 3.

Lemma 3. Let $\left\{b, y_{1}, y_{2}, y_{3}\right\} \subset S^{2}$. If $\left|\left\langle b, y_{1}\right\rangle\right|,\left|\left\langle b, y_{2}\right\rangle\right|$, and $\left|\left\langle b, y_{3}\right\rangle\right|$ are not all equal, then there is a constructible $c \in \mathbb{R}^{3}$ such that

$$
\begin{aligned}
\max & \left\{\left|\left\langle b, y_{k}\right\rangle\right|: k=1,2,3\right\} \\
& >\max \left\{\left|\left\langle\frac{c}{\|c\|}, y_{k}\right\rangle\right|: k=1,2,3\right\} .
\end{aligned}
$$

Furthermore, $\left|\left\langle c /\|c\|, y_{1}\right\rangle\right|=\left|\left\langle c /\|c\|, y_{2}\right\rangle\right|=\left|\left\langle c /\|c\|, y_{3}\right\rangle\right|$. 
Proof

Case 1. $\left\{y_{1}, y_{2}, y_{3}\right\} \subset S^{2}$ is linearly dependent.

Then there are $a_{1}, a_{2}, a_{3} \in \mathbb{R}^{3}$ with at least one (actually two) $a_{k} \neq 0$ such that

$$
a_{1} y_{1}+a_{2} y_{2}+a_{3} y_{3}=0
$$

Therefore, $\operatorname{dim}(\operatorname{ker} Y) \geq 1$, where $Y$ is a $3 \times 3$ matrix with columns $y_{j}$. Hence, $\operatorname{dim}(\operatorname{span} Y)=\operatorname{rank} Y \leq 2$. We can choose $c \in(\operatorname{span} Y)^{\perp}$, so that

$$
\forall k=1,2,3, \quad\left|\left\langle b, y_{k}\right\rangle\right|>0=\left|\left\langle\frac{c}{\|c\|}, y_{k}\right\rangle\right|
$$

since by assumption we know that the $\left|\left\langle b, y_{k}\right\rangle\right|$ cannot all be equal, and hence cannot all equal zero.

Case 2. $\left\{y_{1}, y_{2}, y_{3}\right\} \subset S^{2}$ is linearly independent.

Let $Y$ be the $3 \times 3$ matrix whose columns are $y_{j}$. Then $Y^{T}$ is invertible. Let $c_{1}, \ldots, c_{4}$ be the columns of the following $3 \times 4$ matrix product:

$$
\left[\begin{array}{cccc}
\mid & \mid & \mid & \mid \\
c_{1} & c_{2} & c_{3} & c_{4} \\
\mid & \mid & \mid & \mid
\end{array}\right]=\left(Y^{T}\right)^{-1}\left[\begin{array}{rrrr}
1 & -1 & 1 & 1 \\
1 & 1 & -1 & 1 \\
1 & 1 & 1 & -1
\end{array}\right]
$$

see Figure 3. Notice that

$$
\begin{aligned}
c_{1} & =\left(Y^{T}\right)^{-1}\left[\begin{array}{l}
1 \\
1 \\
1
\end{array}\right] \\
& =\left(Y^{T}\right)^{-1}\left(\left[\begin{array}{r}
-1 \\
1 \\
1
\end{array}\right]+\left[\begin{array}{r}
1 \\
-1 \\
1
\end{array}\right]+\left[\begin{array}{r}
1 \\
1 \\
-1
\end{array}\right]\right) \\
& =c_{2}+c_{3}+c_{4} .
\end{aligned}
$$

Let $c \in\left\{c_{1}, \ldots, c_{4}\right\}$ have the property that $\|c\|=$ $\max \left\{\left\|c_{1}\right\|, \ldots,\left\|c_{4}\right\|\right\}$. We now show that this procedure of matrix multiplication followed by taking the maximum gives rise to a vector $c \in \mathbb{R}^{3}$ which satisfies the conclusion of Lemma 3.

For $j=1,2,3$, define $v_{j}=c_{j+1}-c_{1}$, and set

$$
\begin{aligned}
& Q=\left\{c_{1}+\sum_{j=1}^{3} s_{j} v_{j}: s_{j} \in[0,1]\right\}, \\
& C=\left\{c_{1}+\sum_{j=1}^{3} \varepsilon_{j} v_{j}: \varepsilon_{j} \in\{0,1\}\right\} .
\end{aligned}
$$

Identify a point in $C$ with a vector $\left(\varepsilon_{1}, \varepsilon_{2}, \varepsilon_{3}\right)$. For example, if $\left(\varepsilon_{1}, \varepsilon_{2}, \varepsilon_{3}\right)=(1,0,1)$, then $v=c_{1}+v_{1}+v_{3}$. Observe that since

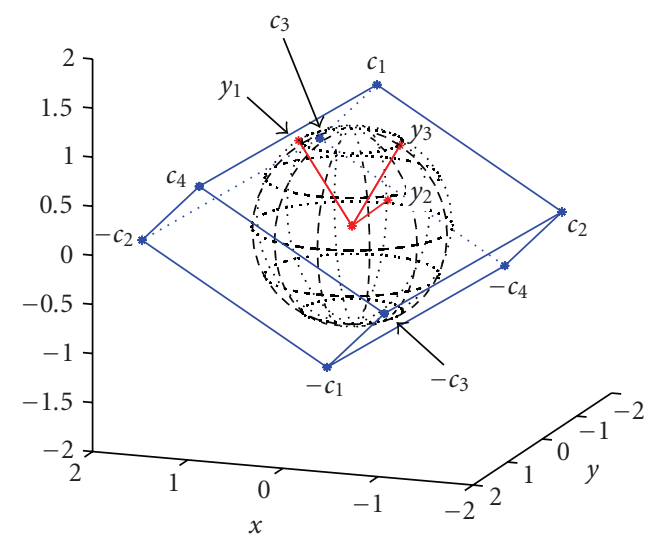

Figure 3: An example showing the points $\pm c_{k}, k=1, \ldots, 4$, and their relationship to the vectors $y_{1}, y_{2}, y_{3}$. Note, $y_{2}$ lies on the plane with vertices $\left\{c_{1}, c_{2},-c_{3}, c_{4}\right\}$.

$c_{1}=c_{2}+c_{3}+c_{4}$, we have the following bijection between $C$ and $\left\{ \pm c_{1}, \pm c_{2}, \pm c_{3}, \pm c_{4}\right\}$ :

$$
\begin{aligned}
& (0,0,0) \longleftrightarrow c_{1}, \\
& (1,0,0) \longleftrightarrow c_{2}, \\
& (0,1,0) \longleftrightarrow c_{3}, \\
& (0,0,1) \longleftrightarrow c_{4}, \\
& (1,1,0) \longleftrightarrow-c_{4}, \\
& (1,0,1) \longleftrightarrow-c_{3}, \\
& (0,1,1) \longleftrightarrow-c_{2}, \\
& (1,1,1) \longleftrightarrow-c_{1} .
\end{aligned}
$$

In particular, $\|c\|=\max \left\{\left\|c^{\prime}\right\|: c^{\prime} \in C\right\}$.

Now, if

$$
H=\left\{v \in \mathbb{R}^{3}:\left|\left\langle v, y_{k}\right\rangle\right| \leq 1 \text { for } k=1,2,3\right\}
$$

then $Q=H$. To see this, we check both containments, but first note that (41) with $j=2$ implies

$$
Y^{T} c_{2}=\left[\begin{array}{r}
-1 \\
1 \\
1
\end{array}\right] \Longrightarrow\left[\begin{array}{l}
y_{1}^{T} c_{2} \\
y_{2}^{T} c_{2} \\
y_{3}^{T} c_{2}
\end{array}\right]=\left[\begin{array}{r}
-1 \\
1 \\
1
\end{array}\right],
$$

that is, $\left\langle y_{1}, c_{2}\right\rangle=-1,\left\langle y_{2}, c_{2}\right\rangle=1$, and $\left\langle y_{3}, c_{2}\right\rangle=1$, and similarly for the other $c_{j}$.

$Q \subset H$. Let $v \in Q$. Then

$$
\begin{aligned}
\left|\left\langle v, y_{k}\right\rangle\right| & =\left|\left\langle c_{1}, y_{k}\right\rangle+\sum_{j=1}^{3} s_{j}\left\langle v_{j}, y_{k}\right\rangle\right| \\
& =\left|1+\sum_{j=1}^{3} s_{j}\left\langle v_{j}, y_{k}\right\rangle\right|
\end{aligned}
$$


whereas

$$
\left\langle v_{j}, y_{k}\right\rangle=\left\langle c_{j+1}-c_{1}, y_{k}\right\rangle= \begin{cases}1-1, & \text { if } k=j \\ -1-1, & \text { if } k \neq j\end{cases}
$$

Thus, $\left|\left\langle v_{j}, y_{k}\right\rangle\right|=\left|1-2 s_{k}\right|$, and so $s_{k} \in[0,1]$ implies that $1-2 s_{k} \in[-1,0]$, and hence $\left|\left\langle v_{j}, y_{k}\right\rangle\right| \leq 1$.

$H \subset Q$. Equivalently, we prove the inclusion $Q^{c} \subset H^{c}$ of complements. Let $v \notin Q$. First, $v_{1}, v_{2}, v_{3}$ are the images of $(-2,0,0)^{T},(0,-2,0)^{T},(0,0,-2)^{T}$, respectively, under the transformation $\left(Y^{T}\right)^{-1}$. Hence, $\left\{v_{1}, v_{2}, v_{3}\right\}$ is a basis for $\mathbb{R}^{3}$. Thus, there are unique elements $s_{1}, s_{2}, s_{3} \in \mathbb{R}^{3}$ such that $v-$ $c_{1}=s_{1} v_{1}+s_{2} v_{2}+s_{3} v_{3}$, that is,

$$
v=c_{1}+s_{1} v_{1}+s_{2} v_{2}+s_{3} v_{3}
$$

Because of the uniqueness of the $s_{j} s, v \notin Q$ implies there is a $j_{0} \in\{1,2,3\}$ such that $s_{j_{0}} \notin[0,1]$. Now, $\left|\left\langle v, y_{j_{0}}\right\rangle\right|=$ $\left|1-2 s_{j_{0}}\right|$, and so $s_{j_{0}} \notin[0,1]$ implies

$$
1-2 s_{j_{0}} \in(-\infty,-1) \cup(1, \infty)
$$

Thus, $\left|\left\langle v, y_{j_{0}}\right\rangle\right|>1$, and so $v \notin H$. Therefore, we have proven $H \subset Q$.

We complete the proof of Lemma 3 as follows. For the set $\left\{b, y_{1}, y_{2}, y_{3}\right\} \subset S^{2}$, let

$$
\left|\left\langle b, y_{k_{b}}\right\rangle\right|=\max \left\{\left|\left\langle b, y_{1}\right\rangle\right|,\left|\left\langle b, y_{2}\right\rangle\right|,\left|\left\langle b, y_{3}\right\rangle\right|\right\},
$$

and set $\lambda_{b}=\left\langle b, y_{k_{b}}\right\rangle$. Then, for any $k=1,2,3$,

$$
\left|\left\langle\frac{b}{\lambda_{b}}, y_{k}\right\rangle\right|=\frac{\left|\left\langle b, y_{k}\right\rangle\right|}{\left|\left\langle b, y_{k_{b}}\right\rangle\right|} \leq \frac{\left|\left\langle b, y_{k_{b}}\right\rangle\right|}{\left|\left\langle b, y_{k_{b}}\right\rangle\right|}=1,
$$

and so $b / \lambda_{b} \in Q$. Now $v \in C$ implies that the elements of $\left\{\left|\left\langle v, y_{k}\right\rangle\right|: k=1,2,3\right\}$ are all equal. By the contrapositive of this implication, we see that the assumption that the elements of $\left\{\left|\left\langle b, y_{k}\right\rangle\right|: k=1,2,3\right\}$ are not all equal implies $b / \lambda_{b} \notin C$. Thus, we have shown $b / \lambda_{b} \in Q \backslash C$. Therefore, by Lemma 2, we have $\left\|b / \lambda_{b}\right\|<\|c\|$; and hence $b \in S^{2}$ implies

$$
\frac{1}{\left|\lambda_{b}\right|}=\frac{1}{\left|\lambda_{b}\right|}\|b\|=\left\|\frac{b}{\lambda_{b}}\right\|<\|c\|
$$

We conclude that

$$
\begin{aligned}
\max & \left\{\left|\left\langle b, y_{k}\right\rangle\right|: k=1,2,3\right\}=\left|\lambda_{b}\right| \\
> & \frac{1}{\|c\|}=\max \left\{\left|\left\langle\frac{c}{\|c\|}, y_{k}\right\rangle\right|: k=1,2,3\right\},
\end{aligned}
$$

and so Lemma 3 is proved.

We can now prove the following theorem.

Theorem 4 ((4,3)-Grassmannian). Let $U=\left\{u_{1}, u_{2}, u_{3}, u_{4}\right\} \subset$ $S^{2} \subset \mathbb{R}^{3}$. If $U$ is $(4,3)$-Grassmannian, then $U$ is equiangular, that is, $\left|\left\langle u_{k}, u_{l}\right\rangle\right|=\beta \in[0,1]$ whenever $k \neq l$.

Proof. We show the contrapositive of the above implication, namely, we prove that if $U$ is not equiangular, then there is a 4-element set $X \subset S^{2}$ such that

$$
\mathcal{M}_{\infty}(X)<\mathcal{M}_{\infty}(U)
$$

Hence, $U$ does not have minimal maximum correlation, and therefore it is not $(4,3)$-Grassmannian.

Suppose $U=\left\{u_{1}, u_{2}, u_{3}, u_{4}\right\}$ is not equiangular. Then there is an $m_{1} \in\{1,2,3,4\}$ such that, if $k_{1}, k_{2}, k_{3}$ are the remaining indices, we have

$$
\begin{aligned}
& \max \left\{\left|\left\langle u_{m_{1}}, u_{k_{1}}\right\rangle\right|,\left|\left\langle u_{m_{1}}, u_{k_{2}}\right\rangle\right|,\left|\left\langle u_{m_{1}}, u_{k_{3}}\right\rangle\right|\right\} \\
& \quad=\mathcal{M}_{\infty}(U)
\end{aligned}
$$

and

$$
\left|\left\langle u_{m_{1}}, u_{k_{1}}\right\rangle\right|,\left|\left\langle u_{m_{1}}, u_{k_{2}}\right\rangle\right|,\left|\left\langle u_{m_{1}}, u_{k_{3}}\right\rangle\right|
$$

are not all equal. Applying Lemma 3 with $b=u_{m_{1}}$ and $\left\{y_{1}, y_{2}, y_{3}\right\}=\left\{u_{k_{1}}, u_{k_{2}}, u_{k_{3}}\right\}$, there is $c \in \mathbb{R}^{3}$ such that

$$
\begin{aligned}
\max & \left\{\left|\left\langle u_{m_{1}}, u_{k_{i}}\right\rangle\right|: i=1,2,3,\right\} \\
& >\max \left\{\left|\left\langle\frac{c}{\|c\|}, u_{k_{i}}\right\rangle\right|: i=1,2,3,\right\} .
\end{aligned}
$$

Let $x_{m_{1}}=c /\|c\|$, see Step 2 in Figure 4 . Since we have only moved the point $u_{m_{1}}$ to $x_{m_{1}}$, the remaining correlations are unaffected since they do not involve $u_{m_{1}}$. Thus

$$
\begin{aligned}
\mathcal{M}_{\infty}(U) & =\max \left\{\left|\left\langle u_{m_{1}}, u_{k_{i}}\right\rangle\right|: i=1,2,3,\right\} \\
& >\max \left\{\left|\left\langle x_{m_{1}}, u_{k_{i}}\right\rangle\right|: i=1,2,3,\right\}=: \alpha .
\end{aligned}
$$

Now either $\mathcal{M}_{\infty}\left(\left\{x_{m_{1}}, u_{k_{1}}, u_{k_{2}}, u_{k_{3}}\right\}\right)=\alpha$, or there is an $m_{2} \in\{1,2,3,4\} \backslash\left\{m_{1}\right\}$ such that if $m_{1}, j_{1}, j_{2}$ are the remaining indices, then

$$
\mathcal{M}_{\infty}(U)=\max \left\{\left|\left\langle u_{m_{2}}, u_{j_{1}}\right\rangle\right|,\left|\left\langle u_{m_{2}}, u_{j_{2}}\right\rangle\right|\right\}
$$

and

$$
\left|\left\langle u_{m_{2}}, x_{m_{1}}\right\rangle\right|,\left|\left\langle u_{m_{2}}, u_{j_{1}}\right\rangle\right|,\left|\left\langle u_{m_{2}}, u_{j_{2}}\right\rangle\right|
$$

are not all equal, where (61) follows from (59). In this case, we apply Lemma 3 to $b=u_{m_{2}}$ and $\left\{y_{1}, y_{2}, y_{3}\right\}=$ $\left\{u_{j_{1}}, u_{j_{2}}, x_{m_{1}}\right\}$. Thus, there is a $c^{\prime} \in \mathbb{R}^{3}$ such that

$$
\begin{aligned}
& \max \left\{\left|\left\langle u_{m_{2}}, x_{m_{1}}\right\rangle\right|,\left|\left\langle u_{m_{2}}, u_{j_{1}}\right\rangle\right|,\left|\left\langle u_{m_{2}}, u_{j_{2}}\right\rangle\right|\right\} \\
&= \max \left\{\left|\left\langle u_{m_{2}}, u_{j_{1}}\right\rangle\right|,\left|\left\langle u_{m_{2}}, u_{j_{2}}\right\rangle\right|\right\} \\
&>\max \left\{\left|\left\langle\frac{c^{\prime}}{\left\|c^{\prime}\right\|}, x_{m_{1}}\right\rangle\right|,\left|\left\langle\frac{c^{\prime}}{\left\|c^{\prime}\right\|}, u_{j_{1}}\right\rangle\right|,\right. \\
&\left.\left|\left\langle\frac{c^{\prime}}{\left\|c^{\prime}\right\|}, u_{j_{2}}\right\rangle\right|\right\} .
\end{aligned}
$$

Let $x_{m_{2}}=c^{\prime} /\left\|c^{\prime}\right\|$, see Step 3 in Figure 4 . Thus

$$
\begin{aligned}
& \mathcal{M}_{\infty}(U) \\
& \quad=\max \left\{\left|\left\langle u_{m_{2}}, u_{j_{1}}\right\rangle\right|,\left|\left\langle u_{m_{2}}, u_{j_{2}}\right\rangle\right|\right\} \\
& \quad>\max \left\{\left|\left\langle x_{m_{2}}, x_{m_{1}}\right\rangle\right|,\left|\left\langle x_{m_{2}}, u_{j_{1}}\right\rangle\right|,\left|\left\langle x_{m_{2}}, u_{j_{2}}\right\rangle\right|\right\} \\
& \quad=: \alpha^{\prime}
\end{aligned}
$$


Therefore, (59) and (63) imply

$$
\begin{aligned}
\mathcal{M}_{\infty}(U) & >\max \left\{\begin{array}{cc}
\left|\left\langle x_{m_{1}}, u_{j_{1}}\right\rangle\right|, & \left|\left\langle x_{m_{1}}, u_{j_{2}}\right\rangle\right|, \\
\left|\left\langle x_{m_{2}}, x_{m_{1}}\right\rangle\right|, & \left|\left\langle x_{m_{2}}, u_{j_{1}}\right\rangle\right|, \\
\left|\left\langle x_{m_{2}}, u_{j_{2}}\right\rangle\right| &
\end{array}\right\} \\
& =\max \left\{\alpha, \alpha^{\prime}\right\},
\end{aligned}
$$

because $j_{1}, j_{2} \in\left\{k_{1}, k_{2}, k_{3}\right\}$.

So either $\mathcal{M}_{\infty}\left(\left\{x_{m_{1}}, x_{m_{2}}, u_{j_{1}}, u_{j_{2}}\right\}\right)=\max \left\{\alpha, \alpha^{\prime}\right\}$ or else $\mathcal{M}_{\infty}(U)=\left|\left\langle u_{j_{1}}, u_{j_{2}}\right\rangle\right|$.

In the latter case, (63) implies that $\left|\left\langle u_{j_{1}}, u_{j_{2}}\right\rangle\right|, \mid\left\langle u_{j_{1}}\right.$, $\left.x_{m_{1}}\right\rangle||,\left\langle u_{j_{1}}, x_{m_{2}}\right\rangle \mid$ are not all equal, and so we apply Lemma 3 to $b=u_{j_{1}}$, and $\left\{y_{1}, y_{2}, y_{3}\right\}=\left\{u_{j_{2}}, x_{m_{1}}, x_{m_{2}}\right\}$. Thus, there is $c^{\prime \prime} \in \mathbb{R}^{3}$ such that

$$
\begin{aligned}
\max & \left\{\left|\left\langle u_{j_{1}}, x_{m_{1}}\right\rangle\right|,\left|\left\langle u_{j_{1}}, x_{m_{2}}\right\rangle\right|,\left|\left\langle u_{j_{1}}, u_{j_{2}}\right\rangle\right|\right\} \\
& =\left|\left\langle u_{j_{2}}, u_{j_{1}}\right\rangle\right| \\
& >\max \left\{\begin{array}{c}
\left|\left\langle\frac{c^{\prime \prime}}{\left\|c^{\prime \prime}\right\|}, x_{m_{1}}\right\rangle\right|,\left|\left\langle\frac{c^{\prime \prime}}{\left\|c^{\prime \prime}\right\|}, x_{m_{2}}\right\rangle\right|, \\
\left|\left\langle\frac{c^{\prime \prime}}{\left\|c^{\prime \prime}\right\|}, u_{j_{2}}\right\rangle\right|
\end{array}\right\} .
\end{aligned}
$$

Let $x_{m_{3}}=c^{\prime \prime} /\left\|c^{\prime \prime}\right\|$ and let $X=\left\{x_{m_{1}}, x_{m_{2}}, x_{m_{3}}, u_{j_{2}}\right\}$. Then (64) and (65) imply

$$
\begin{aligned}
& \mathcal{M}_{\infty}(U) \\
& >\max \left\{\begin{array}{lll}
\left|\left\langle x_{m_{3}}, x_{m_{1}}\right\rangle\right|, & \left|\left\langle x_{m_{3}}, x_{m_{2}}\right\rangle\right|, & \left|\left\langle x_{m_{3}}, u_{j_{2}}\right\rangle\right|, \\
\left|\left\langle x_{m_{2}}, x_{m_{1}}\right\rangle\right|, & \left|\left\langle x_{m_{2}}, u_{j_{2}}\right\rangle\right|, & \left|\left\langle x_{m_{1}}, u_{j_{2}}\right\rangle\right|
\end{array}\right\} \\
& =\mathcal{M}_{\infty}(X) .
\end{aligned}
$$

Next we show that if a four-element set is equiangular, then the vectors are parallel to the diagonals of a cube or to four of the diagonals of an icosahedron.

Theorem 5. If $u_{1}, u_{2}, u_{3}, u_{4} \in S^{2}$ and $\left|\left\langle u_{k}, u_{l}\right\rangle\right|=\alpha$ for $k, l \in$ $\{1, \ldots, 4\}$ with $k \neq l$, then

$$
\alpha=\frac{1}{3} \quad \text { or } \quad \alpha=\frac{1}{\sqrt{5}}
$$

Proof. Since sign changes and rotations do not effect inner products, let $P$ be an element of $S_{3}$ which rotates $u_{1}$ to $\delta_{3}=$ $(1,0,0)^{T}$. For $k=1,2,3,4$, let

$$
\varepsilon_{k}=\operatorname{sign}\left\langle P x_{k}, \delta_{3}\right\rangle \text {, }
$$

and let $Q \in S_{3}$ so that $Q$ fixes $\delta_{3}$ and $Q$ rotates $\varepsilon_{k} P x_{2}$ to the positive $x z$-plane, that is, $\left\langle Q \varepsilon_{k} P x_{2}, \delta_{1}\right\rangle \geq 0$ and $\left\langle Q \varepsilon_{k} P x_{2}, \delta_{2}\right\rangle=0$. Then, for $k \neq l$,

$$
\alpha=\left|\left\langle u_{k}, u_{l}\right\rangle\right|=\left|\left\langle\varepsilon_{k} Q P u_{k}, \varepsilon_{l} Q P u_{l}\right\rangle\right|=\left|\left\langle w_{k}, w_{l}\right\rangle\right|,
$$

where $w_{k}=\varepsilon_{k} Q P u_{k}$. By the choice of $\varepsilon_{k}$, for $k=2,3,4$, we have

$$
\alpha=\left|\left\langle w_{1}, w_{k}\right\rangle\right|=\left\langle\delta_{3}, w_{k}\right\rangle
$$

so that the third component of $w_{k}$ is $\alpha$. Also, $0=\left\langle\delta_{2}, w_{2}\right\rangle$, and $w_{2} \in S^{2}$, so that the first component of $w_{2}$ is $\sqrt{1-\alpha^{2}}$. Therefore, we have

$$
\begin{aligned}
& w_{1}=(0,0,1)^{T}, \\
& w_{2}=\left(\sqrt{1-\alpha^{2}}, 0, \alpha\right)^{T}, \\
& w_{3}=\left(x_{3}, y_{3}, \alpha\right)^{T}, \\
& w_{4}=\left(x_{4}, y_{4}, \alpha\right)^{T} .
\end{aligned}
$$

We now have four cases (see Table 3) where both Cases 2 and 3 have three subcases which, by relabeling, can be reduced to the considered case.

Case 1. For $k=3,4,\left\langle w_{2}, w_{k}\right\rangle=\alpha$ implies

$$
x_{k}=\frac{\alpha-\alpha^{2}}{\sqrt{1-\alpha^{2}}}=\alpha \sqrt{\frac{1-\alpha}{1+\alpha}} .
$$

Then (72) and $\left\|w_{k}\right\|^{2}=1$ imply

$$
y_{k}= \pm \sqrt{\frac{1+\alpha-2 \alpha^{2}}{1+\alpha}} .
$$

In addition, (72) and $\left\langle w_{3}, w_{4}\right\rangle=\alpha$ imply

$$
y_{3} \cdot y_{4}=\frac{\alpha(1-\alpha)}{1+\alpha} .
$$

Combining (73) and (74), we have

$$
\begin{aligned}
-\frac{1+\alpha-2 \alpha^{2}}{1+\alpha}=\frac{\alpha(1-\alpha)}{1+\alpha} & \Longrightarrow 2 \alpha^{2}+1=0 \\
& \Longrightarrow \alpha \in \mathbb{C} \backslash \mathbb{R},
\end{aligned}
$$

hence Case 1 is impossible.

Case 2. Now $\left\langle w_{2}, w_{3}\right\rangle=\alpha$ implies

$$
x_{3}=\frac{\alpha-\alpha^{2}}{\sqrt{1-\alpha^{2}}},
$$

and $\left\langle w_{2}, w_{4}\right\rangle=-\alpha$ implies

$$
x_{4}=\frac{-\alpha-\alpha^{2}}{\sqrt{1-\alpha^{2}}},
$$

and (76) and (77) imply

$$
y_{3} \cdot y_{4}=\alpha
$$

Then, (76) and $\left\|w_{3}\right\|^{2}=1$ imply

$$
y_{3}^{2}=-\frac{2 \alpha^{2}-\alpha-1}{\alpha+1}=\frac{(2 \alpha+1)(\alpha-1)}{\alpha+1},
$$

and (77) and $\left\|w_{4}\right\|^{2}=1$ imply

$$
y_{4}^{2}=\frac{2 \alpha^{2}+\alpha-1}{\alpha-1}=\frac{(2 \alpha-1)(\alpha+1)}{\alpha-1} .
$$

Finally, (78), (79), and (80) imply

$$
-\alpha^{2}=(2 \alpha+1)(2 \alpha-1) \Longrightarrow \alpha= \pm \frac{1}{\sqrt{5}} .
$$




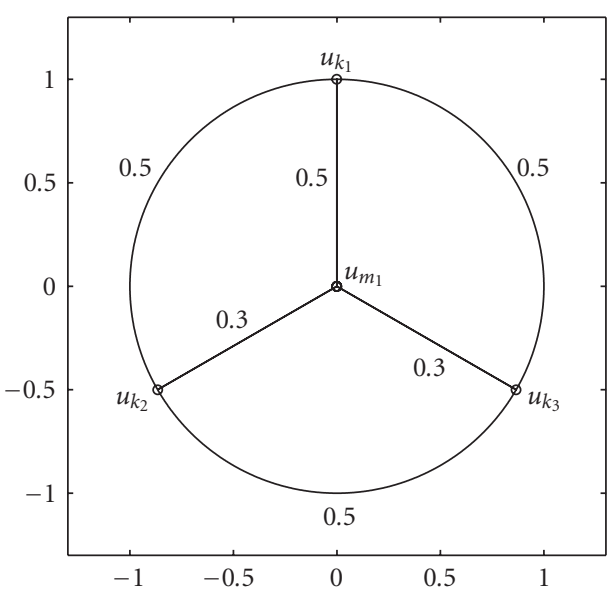

(a)

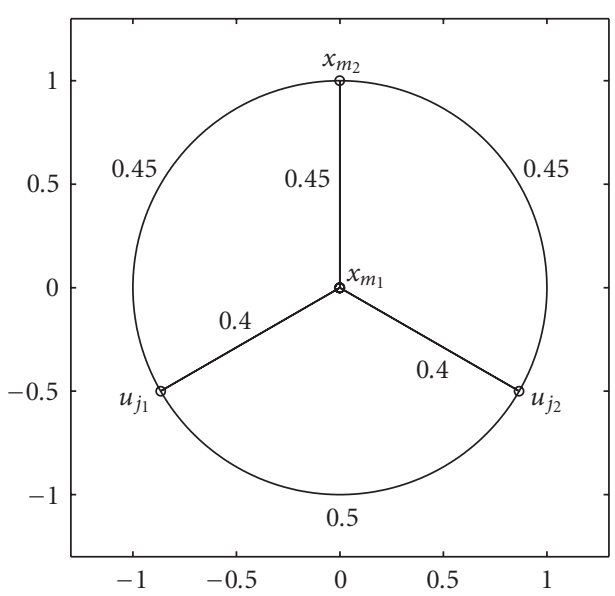

(c)

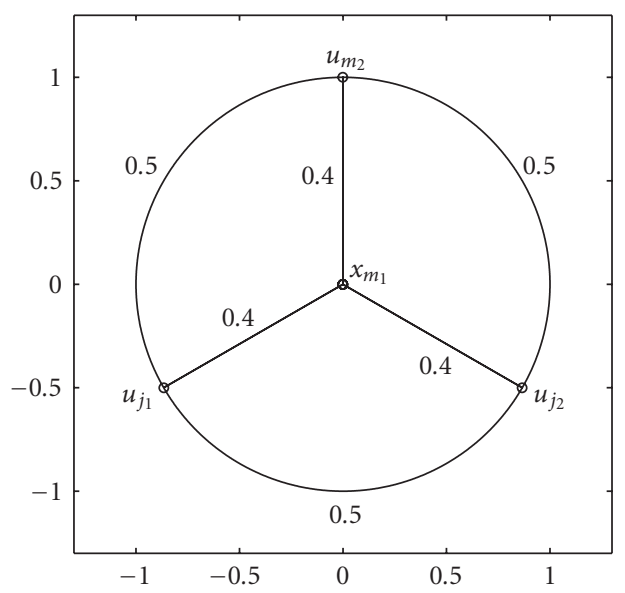

(b)

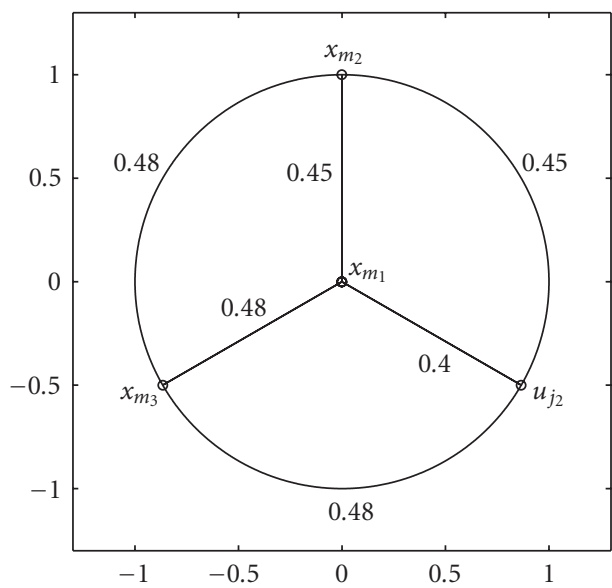

(d)

FIgURE 4: An example of the four steps in proving Theorem 4. A number next to an edge represents the inner product of the two boundary points of the edge. (a) Step 1, (b) Step 2, (c) Step 3, and (d) Step 4.

TABLE 3: Four main cases in the proof of Theorem 5.

\begin{tabular}{lcccc}
\hline & $\left\langle w_{2}, w_{3}\right\rangle$ & $\left\langle w_{2}, w_{4}\right\rangle$ & $\left\langle w_{3}, w_{4}\right\rangle$ & \\
\hline Case 1 & $\alpha$ & $\alpha$ & $\alpha$ & Impossible \\
Case 2 & $-\alpha$ & $\alpha$ & $\alpha$ & $\alpha=1 / \sqrt{5}$ \\
Case 3 & $-\alpha$ & $-\alpha$ & $\alpha$ & $\alpha=1 / \sqrt{5}$ \\
Case 4 & $-\alpha$ & $-\alpha$ & $-\alpha$ & $\alpha=1 / 3$ \\
\hline
\end{tabular}

Since $\alpha$ is assumed to be positive, we have proven Case 2.

Case 3. For $k=3,4,\left\langle w_{2}, w_{k}\right\rangle=-\alpha$ implies

$$
x_{k}=\frac{-\alpha-\alpha^{2}}{\sqrt{1-\alpha^{2}}}=-\alpha \sqrt{\frac{1+\alpha}{1-\alpha}} .
$$

Then (82) and $\left\|w_{k}\right\|^{2}=1$ imply

$$
y_{k}^{2}=\frac{2 \alpha^{2}+\alpha-1}{\alpha-1}=\frac{(2 \alpha-1)(\alpha+1)}{\alpha-1} \text {. }
$$

Hence, (82) and $\left\langle w_{3}, w_{4}\right\rangle=\alpha$ imply

$$
y_{3} \cdot y_{4}=\frac{\alpha-3 \alpha^{2}}{1-\alpha}
$$

Combining (83) and (84), we have

$$
\begin{aligned}
& -\frac{(2 \alpha-1)(\alpha+1)}{\alpha-1}=-y_{k}^{2}=y_{3} \cdot y_{4}=\frac{\alpha-3 \alpha^{2}}{1-\alpha} \\
& \Longrightarrow \alpha= \pm \frac{1}{\sqrt{5}},
\end{aligned}
$$

and since $\alpha$ is positive, we have proven Case 3 .

Case 4. This is the same as Case 3 except that $\left\langle w_{3}, w_{4}\right\rangle=\alpha$ and (82) imply

$$
y_{3} \cdot y_{4}=\frac{\alpha(\alpha+1)}{\alpha-1}
$$


Combining (83) and (86), we have

$$
\begin{aligned}
& -\frac{(2 \alpha-1)(\alpha+1)}{\alpha-1}=-y_{k}^{2}=y_{3} \cdot y_{4}=\frac{\alpha(\alpha+1)}{\alpha-1} \\
& \Longrightarrow \alpha=\frac{1}{3},
\end{aligned}
$$

and we have proven Case 4.

Therefore, the theorem is proved.

By Theorem 5 , and since $1 / \sqrt{5}>1 / 3$, we see that the $(4,3)$-Grassmannian bound is $1 / 3$, which is also seen to be optimal by inspection.

\section{6. (5, 3)-GRASSMANNIAN FRAMES}

We first introduce some ideas from convex analysis [17-19].

Definition 4. A set $A \subset \mathbb{R}^{n}$ is convex if for any $x_{1}, x_{2} \in A$, and for any $\lambda \in[0,1]$,

$$
\lambda x_{1}+(1-\lambda) x_{2} \in A .
$$

A point $x \in A$ is an extreme point of $A$ if whenever $x=\lambda x_{1}+$ $(1-\lambda) x_{2}$, where $0<\lambda<1$ and $x_{1}, x_{2} \in A$, then $x=x_{1}=x_{2}$. Given a set $A \subset \mathbb{R}^{n}$, the convex hull of $A$ is

$\operatorname{Hull}(A)$

$$
=\left\{\sum_{j=1}^{m} \lambda_{j} x_{j}: \sum_{j=1}^{m} \lambda_{j}=1, \lambda_{j}>0, x_{j} \in A, m \in \mathbb{N}\right\} .
$$

There is the following relationship between extreme points, convex hulls, and convex sets, [20].

Theorem 6. A nonempty bounded convex set in $\mathbb{R}^{d}$ is the convex hull of its set of extreme points.

We need the following two convexity propositions to prove Lemma 4, which in turn is used to prove Lemma 5, the key lemma for computing the $(5,3)$-Grassmannian bound in Theorem 7.

Proposition 2. Let $N \geq d$, let $Y=\left\{y_{1}, \ldots, y_{N}\right\} \subset S^{d-1} \subset \mathbb{R}^{d}$, and assume $\operatorname{span}(Y)=\mathbb{R}^{d}$. Let

$$
Q=\left\{v \in \mathbb{R}^{d}:\left|\left\langle v, y_{k}\right\rangle\right| \leq 1, \text { for } k=1, \ldots, N\right\}
$$

and let $C$ be the set of extreme points of $Q$. Then

(a) $Q$ is a bounded convex set,

(b) if $v_{0} \in C$, then there are at least $d$ distinct integers $k_{1}, \ldots, k_{d} \in\{1, \ldots, N\}$ such that $\left|\left\langle v_{0}, y_{k_{i}}\right\rangle\right|=1$ for $i=$ $1, \ldots, d$,

(c) $\operatorname{card}(C) \leq\left(\begin{array}{l}N \\ d\end{array}\right) 2^{d}<\infty$.

Under the same hypotheses of Proposition 2, we have the following result, whose proof is in Appendix C.

Proposition 3. Let $N, d, Y, Q$, and $C$ be as in Proposition 2, and let $c \in C$ have the property that $\|c\|=\max \left\{\left\|c^{\prime}\right\|: c^{\prime} \in\right.$ $C\}$. Then, for any $v \in Q \backslash C$,

$$
\|v\|<\|c\| .
$$

Following the basic geometric idea in [10], we can reduce the correlation of a given frame. The following lemma requires the previous propositions as well as Theorem 3.

Lemma 4. Let $U=\left\{b, y_{1}, y_{2}, y_{3}, y_{4}\right\} \subset S^{2} \subset \mathbb{R}^{3}$, and let $\alpha=$ $\mathcal{M}_{\infty}(U)$. Assume $\left|\left\langle b, y_{1}\right\rangle\right|<\alpha$ and $\left|\left\langle b, y_{2}\right\rangle\right|<\alpha$. Then there exists $c \in \mathbb{R}^{3}$ such that

$$
\left|\left\langle\frac{c}{\|c\|}, y_{k}\right\rangle\right|<\alpha \quad \text { for } k=1,2,3,4 .
$$

Lemma 5. Let $U=\left\{u_{1}, \ldots, u_{5}\right\}$ be a $(5,3)$-Grassmannian frame, and let $\alpha=\mathcal{M}_{\infty}(U)$. Then for any $j$, there are distinct $j_{1}, j_{2}, j_{3} \in\{1, \ldots, 5\} \backslash\{j\}$ such that

$$
\left|\left\langle u_{j}, u_{j_{k}}\right\rangle\right|=\alpha \quad \text { for } k=1,2,3 .
$$

Proof. We prove the contrapositive. By relabeling if necessary, without loss of generality, assume $\left|\left\langle u_{1}, u_{2}\right\rangle\right|<\alpha$ and $\left|\left\langle u_{1}, u_{3}\right\rangle\right|<\alpha$. We use Lemma 4 to construct a new set $W$ for which $\mathcal{M}_{\infty}(W)<\alpha$. This shows $U$ is not $(5,3)$ Grassmannian.

First, let $b=u_{1}$ and $\left\{y_{1}, \ldots, y_{4}\right\}=\left\{u_{2}, \ldots, u_{5}\right\}$ and apply Lemma 4. Then there is a $c_{1} \in \mathbb{R}^{3}$ such that

$$
\left|\left\langle\frac{c_{1}}{\left\|c_{1}\right\|}, u_{k}\right\rangle\right|<\alpha \quad \text { for } k=2,3,4,5
$$

Second, consider the set $\tilde{U}:=\left\{u_{2}, \ldots, u_{5}\right\}$. We have two cases,

Case 1. There exist $j_{0}, k_{0} \in\{2,3,4,5\}$ with $j_{0} \neq k_{0}$, for which $\left|\left\langle u_{j_{0}}, u_{k_{0}}\right\rangle\right|<\alpha$.

For ease in notation, by relabeling if necessary, we assume $j_{0}=2$ and $k_{0}=3$. In this case, we can apply Lemma 4 with $b=u_{2}$ and $\left\{y_{1}, \ldots, y_{4}\right\}=\left\{c_{1} /\left\|c_{1}\right\|, u_{3}, u_{4}, u_{5}\right\}$, and construct $c_{2} \in \mathbb{R}^{3}$ such that

$$
\begin{gathered}
\left|\left\langle\frac{c_{1}}{\left\|c_{1}\right\|}, \frac{c_{2}}{\left\|c_{2}\right\|}\right\rangle\right|<\alpha, \\
\max \left\{\left|\left\langle\frac{c_{2}}{\left\|c_{2}\right\|}, u_{k}\right\rangle\right|: k=3,4,5\right\}<\alpha .
\end{gathered}
$$

Now we can apply Lemma 4 to the remaining points and produce a frame with a strictly smaller value of $\mathcal{M}_{\infty}$. In fact, since $\left|\left\langle c_{i} /\left\|c_{i}\right\|, u_{3}\right\rangle\right|<\alpha$ for $i=1,2$, we let $b=u_{3}$ and $\left\{y_{1}, \ldots, y_{4}\right\}=\left\{c_{1} /\left\|c_{1}\right\|, c_{2} /\left\|c_{2}\right\|, u_{4}, u_{5}\right\}$. Then, by Lemma 4 , there is a $c_{3} \in \mathbb{R}^{d}$ such that

$$
\begin{gathered}
\left|\left\langle\frac{c_{3}}{\left\|c_{3}\right\|}, \frac{c_{i}}{\left\|c_{i}\right\|}\right\rangle\right|<\alpha \quad \text { for } i=1,2, \\
\max \left\{\left|\left\langle\frac{c_{3}}{\left\|c_{3}\right\|}, u_{k}\right\rangle\right|: k=4,5\right\}<\alpha .
\end{gathered}
$$

Finally, apply Lemma 4 one last time to $b=u_{4}$ and

$$
\left\{y_{1}, \ldots, y_{4}\right\}=\left\{\frac{c_{1}}{\left\|c_{1}\right\|}, \frac{c_{2}}{\left\|c_{2}\right\|}, \frac{c_{3}}{\left\|c_{3}\right\|}, u_{5}\right\},
$$

and obtain $c_{4} \in \mathbb{R}^{d}$ for which

$$
\begin{gathered}
\left|\left\langle\frac{c_{4}}{\left\|c_{4}\right\|}, \frac{c_{i}}{\left\|c_{i}\right\|}\right\rangle\right|<\alpha \quad \text { for } i=1,2,3, \\
\left|\left\langle\frac{c_{4}}{\left\|c_{4}\right\|}, u_{5}\right\rangle\right|<\alpha .
\end{gathered}
$$


Thus, if we let $W=\left\{c_{1} /\left\|c_{1}\right\|, c_{2} /\left\|c_{2}\right\|, c_{3} /\left\|c_{3}\right\|, c_{4} /\left\|c_{4}\right\|, u_{5}\right\}$, then, by construction, for any $i, j \in\{1, \ldots, 4\}, i \neq j$, we have $\left|\left\langle c_{i} /\left\|c_{i}\right\|, c_{1} /\left\|c_{1}\right\|\right\rangle\right|<\alpha$ and $\left|\left\langle c_{i} /\left\|c_{i}\right\|, u_{5}\right\rangle\right|<\alpha$. Hence,

$$
\mathcal{M}_{\infty}(W)<\alpha=\mathcal{M}_{\infty}(U),
$$

and so $U$ is not $(5,3)$-Grassmannian. This finishes Case 1.

Case 2. $\tilde{U}$ is equiangular.

Since $\tilde{U}$ has four elements, Theorem 5 implies $\alpha=1 / 3$ or $\alpha=1 / \sqrt{5}$. If $\alpha=1 / 3$, then set

$$
\beta=\max \left\{\left|\left\langle\frac{c_{1}}{\left\|c_{1}\right\|}, u_{k}\right\rangle\right|: k=2,3,4,5\right\} .
$$

Thus, by construction of $c_{1}$, we have $\beta<1 / 3$ and

$$
\mathcal{M}_{\infty}\left(\left\{\frac{c_{1}}{\left\|c_{1}\right\|}\right\} \cup \tilde{U}\right)=\max \left\{\frac{1}{3}, \beta\right\}=\frac{1}{3},
$$

whereas Theorem 3 with $N=5$ and $d=3$ implies

$$
\frac{1}{\sqrt{6}} \leq \mathcal{M}_{\infty}\left(\left\{\frac{c_{1}}{\left\|c_{1}\right\|}\right\} \cup \tilde{U}\right)=\frac{1}{3},
$$

a contradiction.

Thus, $\alpha=1 / \sqrt{5}$; and $\left|\left\langle u_{1}, u_{k}\right\rangle\right|<\alpha$ for $k=2,3,4,5$, and $\left|\left\langle u_{k}, u_{j}\right\rangle\right|=\alpha$ for $k \neq j$ and $k, j \in\{2,3,4,5\}$.

We seek to find a contradiction. Without loss of generality, the setting can reduce to the following general position by using rotations and sign changes as in Theorem 5:

$$
\begin{gathered}
u_{2}=(0,0,1)^{T}, \\
u_{3}=\left(\sqrt{1-\alpha^{2}}, 0, \alpha\right)^{T}, \\
u_{4}, u_{5} \in\left\{p_{1}, p_{2}, p_{3}, p_{4}\right\},
\end{gathered}
$$

where

$$
\begin{aligned}
& p_{1}=\left(\alpha \sqrt{\frac{1-\alpha}{1+\alpha}}, \sqrt{\frac{(1+2 \alpha)(1-\alpha)}{1+\alpha}}, \alpha\right)^{T} \\
&=\left(\sqrt{1-\alpha^{2}} \cos \left(\frac{2 \pi}{5}\right), \sqrt{1-\alpha^{2}} \sin \left(\frac{2 \pi}{5}\right), \alpha\right)^{T}, \\
& p_{2}=\left(-\alpha \sqrt{\frac{1+\alpha}{1-\alpha}}, \sqrt{\frac{(1-2 \alpha)(1+\alpha)}{1-\alpha}}, \alpha\right)^{T} \\
&=\left(\sqrt{1-\alpha^{2}} \cos \left(\frac{4 \pi}{5}\right), \sqrt{1-\alpha^{2}} \sin \left(\frac{4 \pi}{5}\right), \alpha\right)^{T}, \\
& p_{3}=\left(-\alpha \sqrt{\frac{1+\alpha}{1-\alpha}},-\sqrt{\frac{(1-2 \alpha)(1+\alpha)}{1-\alpha}}, \alpha\right)^{T} \\
&=\left(\sqrt{1-\alpha^{2}} \cos \left(-\frac{4 \pi}{5}\right),\right. \\
& p_{4}=\left(\sqrt{1-\alpha^{2}} \sin \left(-\frac{4 \pi}{5}\right), \alpha\right)^{T}, \\
&=\left(\sqrt{1-\alpha} \frac{\sqrt{1-\alpha}}{1-\alpha^{2}} \sin \left(-\frac{2 \pi}{5}\right), \alpha\right)^{T} . \\
&\left.\frac{(1+2 \alpha)(1-\alpha)}{1+\alpha}, \alpha\right)^{T}
\end{aligned}
$$

Therefore, if

$$
A=\left(\begin{array}{ccc}
\cos \left(\frac{2 \pi}{5}\right) & -\sin \left(\frac{2 \pi}{5}\right) & 0 \\
\sin \left(\frac{2 \pi}{5}\right) & \cos \left(\frac{2 \pi}{5}\right) & 0 \\
0 & 0 & 1
\end{array}\right)
$$

and $p_{0}=u_{3}$, then

$$
\begin{aligned}
& A^{k}\left(p_{0}, p_{1}, p_{2}, p_{3}, p_{4}\right) \\
& \quad=\left(p_{\sigma(0)}, p_{\sigma(1)}, p_{\sigma(2)}, p_{\sigma(3)}, p_{\sigma(4)}\right),
\end{aligned}
$$

where $\sigma(n)=(n+k)(\bmod 5)$.

If we set $\beta=\left|\left\langle u_{1}, u_{2}\right\rangle\right|<\alpha$, then, by changing the sign of $u_{1}$ if necessary and since $\left\|u_{1}\right\|=1$, we may assume

$$
u_{1}=\left(\sqrt{1-\beta^{2}} \cos t_{0}, \sqrt{1-\beta^{2}} \sin t_{0}, \beta\right)^{T},
$$

for some fixed $t_{0} \in[-\pi, \pi)$. Hence,

$$
\begin{aligned}
& \left|\left\langle u_{1}, u_{3}\right\rangle\right|<\alpha \\
& \Longleftrightarrow\left|\sqrt{1-\alpha^{2}} \sqrt{1-\beta^{2}} \cos t_{0}+\alpha \beta\right|<\alpha \\
& \Longleftrightarrow \frac{-\alpha}{\sqrt{1-\alpha^{2}}} \frac{1+\beta}{\sqrt{1-\beta^{2}}}<\cos t_{0}<\frac{\alpha}{\sqrt{1-\alpha^{2}}} \frac{1-\beta}{\sqrt{1-\beta^{2}}} \\
& \Longleftrightarrow \underbrace{\cos ^{-1}\left(\frac{1}{2} \sqrt{\frac{1-\beta}{1+\beta}}\right)}_{\gamma_{1}(\beta)}<\left|t_{0}\right|<\underbrace{\cos ^{-1}\left(-\frac{1}{2} \sqrt{\frac{1+\beta}{1-\beta}}\right)}_{\gamma_{2}(\beta)} .
\end{aligned}
$$

We observe that

$$
\begin{aligned}
& \gamma_{1}(\beta)= \begin{cases}\frac{6 \pi}{15}=\frac{2 \pi}{5}, & \text { if } \beta=\alpha, \\
\frac{5 \pi}{15}=\frac{\pi}{3}, & \text { if } \beta=0,\end{cases} \\
& \gamma_{2}(\beta)= \begin{cases}\frac{12 \pi}{15}=\frac{4 \pi}{5}, & \text { if } \beta=\alpha, \\
\frac{10 \pi}{15}=\frac{2 \pi}{3}, & \text { if } \beta=0,\end{cases}
\end{aligned}
$$

and that $(d / d \beta)\left(\gamma_{2}-\gamma_{1}\right)(\beta)>0$ for $\beta \in(0, \alpha)$, see Figure 5 . Thus $5 \pi / 15 \leq \gamma_{2}(\beta)-\gamma_{1}(\beta)<6 \pi / 15$, when $\beta \in[0, \alpha)$.

Hence, for a fixed $\beta \in[0, \alpha)$,

$$
\gamma_{2}(\beta)<\gamma_{1}(\beta)+\frac{6 \pi}{15}
$$

and, for $k=1,2,3,4$, we have

$$
\begin{aligned}
\alpha & >\left|\left\langle u_{1}, p_{k}\right\rangle\right|=\left|\left\langle A^{-k} u_{1}, A^{-k} p_{k}\right\rangle\right| \\
& =\left|\left\langle A^{-k} u_{1}, p_{0}\right\rangle\right|=\left|\left\langle A^{-k} u_{1}, u_{3}\right\rangle\right|
\end{aligned}
$$

where

$$
\begin{aligned}
A^{-k} u_{1}= & \left(\sqrt{1-\beta^{2}} \cos \left(t_{0}-\frac{2 \pi k}{5}\right),\right. \\
& \left.\sqrt{1-\beta^{2}} \sin \left(t_{0}-\frac{2 \pi k}{5}\right), \beta\right)^{T} .
\end{aligned}
$$




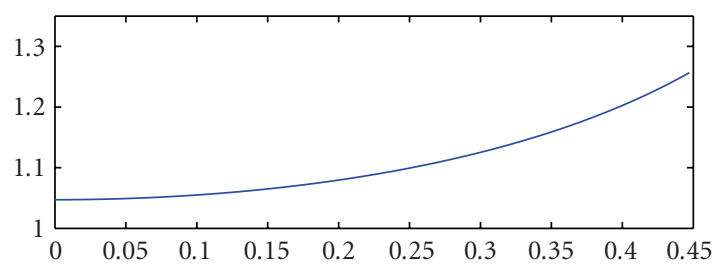

(a)

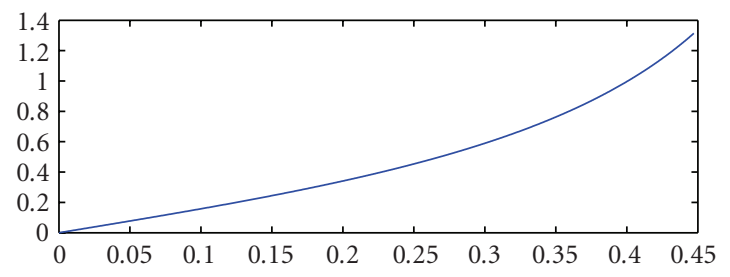

(b)

Figure 5: (a) The function $\gamma_{2}-\gamma_{1}$. (b) The function $(d / d \beta)\left(\gamma_{2}-\gamma_{1}\right)$. We can see that the original function is strictly increasing.

Therefore, by (111),

$$
\alpha>\left|\left\langle u_{1}, p_{k}\right\rangle\right| \Longleftrightarrow \gamma_{1}(\beta) \leq\left|t_{0}-\frac{2 \pi k}{5}\right| \leq \gamma_{2}(\beta),
$$

for $k=0,1,2,3,4$. These inequalities define ten intervals on the torus $\mathbb{T}_{2 \pi}$. If we plot these ten intervals on $\mathbb{T}_{2 \pi}$, we note that no set of three of them overlaps, see Figure 6 . This assertion can also be seen since

$$
\begin{aligned}
& \gamma_{1}(\beta) \leq\left|t_{0}-\frac{2 \pi k}{5}\right| \leq \gamma_{2}(\beta) \\
& \Longrightarrow \gamma_{1}(\beta) \leq\left|t_{0}-\frac{2 \pi k}{5}\right|<\gamma_{1}(\beta)+\frac{2 \pi}{5} \\
& \Longrightarrow t_{0} \in \underbrace{[\gamma+\varepsilon k, \gamma+\varepsilon(k+1))}_{P_{k}} \\
& \cup \underbrace{[-\gamma+\varepsilon(k-1),-\gamma+\varepsilon k)}_{N_{k}},
\end{aligned}
$$

where $\gamma=\gamma_{1}(\beta), \varepsilon=2 \pi / 5$, and $k=0,1,2,3,4$. Thus, $\bigcup_{k=0}^{4} P_{k}$ is a disjoint cover of $\mathbb{T}_{2 \pi} \backslash[\gamma-\varepsilon, \gamma)$, and $\bigcup_{k=0}^{4} N_{k}$ is a disjoint cover of $\mathbb{T}_{2 \pi} \backslash[-\gamma+\varepsilon,-\gamma)$. Hence, $t_{0}$ can be in at most two of the ten sets $P_{k}, N_{k}$.

Now, by assumption, $\left|\left\langle u_{1}, u_{3}\right\rangle\right|=\left|\left\langle u_{1}, p_{0}\right\rangle\right|<\alpha$. Also $\left|\left\langle u_{1}, u_{4}\right\rangle\right|<\alpha$ and $\left|\left\langle u_{1}, u_{5}\right\rangle\right|<\alpha$, where $u_{4}, u_{5} \in$ $\left\{p_{1}, p_{2}, p_{3}, p_{4}\right\}$. Thus, (116) implies $t_{0}$ lies in three of the ten intervals represented in Figure 6, a contradiction. Consequently, $\tilde{U}$ cannot be equiangular.

Finally, using Lemma 5, we have the following result.

Theorem 7. If $U \subset S^{2} \subset \mathbb{R}^{3}$ is (5,3)-Grassmannian, then $\mathcal{M}_{\infty}(U)=1 / \sqrt{5}$.

Proof. Let $\alpha=\mathcal{M}_{\infty}(U)$, and consider the graph whose vertices are $u_{1}, \ldots, u_{5}$, and whose edges are defined as follows:

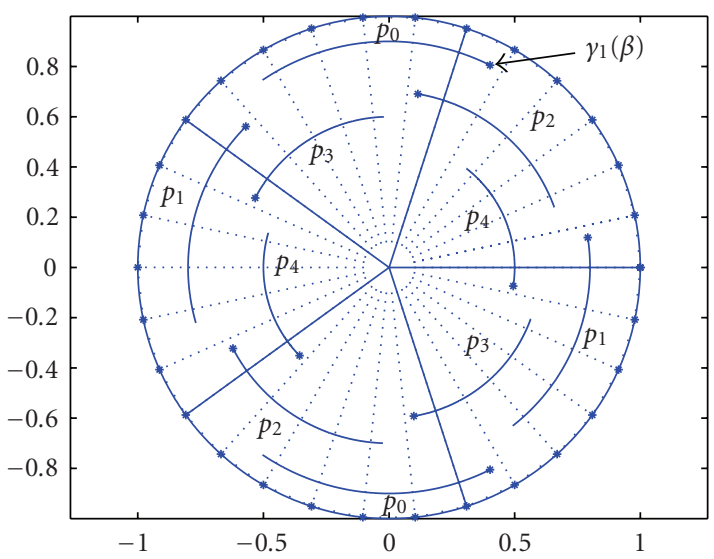

Figure 6: Ten intervals on $\mathbb{T}_{2 \pi}$ corresponding to the points $p_{0}=u_{3}$, and $p_{1}, \ldots, p_{4}$.

for any pair of points $u_{k}, u_{j} \in U$ with $k \neq j$, an edge connects $u_{k}$ and $u_{j}$ if and only if $\left|\left\langle u_{k}, u_{j}\right\rangle\right|=\alpha$. We call the number of edges emanating from a vertex $u_{k}$, the degree of $u_{k}$, denoted by $\operatorname{deg}\left(u_{k}\right)$. Then Lemma 5 implies that

$$
\sum_{k=1}^{5} \operatorname{deg}\left(u_{k}\right) \geq \sum_{k=1}^{5} 3=15 .
$$

Since each edge connects two vertices, the sum of the degrees must be an even number. Thus, at least one vertex $u_{j}$ must have degree 4 , that is, there is a $j \in\{1, \ldots, 5\}$, such that $\left|\left\langle u_{j}, u_{j_{i}}\right\rangle\right|=\alpha$ for $i=1, \ldots, 4$, where $\left\{j_{1}, j_{2}, j_{3}, j_{4}\right\}=$ $\{1,2,3,4,5\} \backslash\{j\}$. By relabeling if necessary, we may assume

$$
\begin{array}{ll}
\left|\left\langle u_{1}, u_{k}\right\rangle\right|=\alpha & \text { for } k=2,3,4,5, \\
\left|\left\langle u_{2}, u_{k}\right\rangle\right|=\alpha & \text { for } k=3,4 .
\end{array}
$$

Furthermore, we can reduce to the general position used in Theorem 5, that is, assume

$$
\begin{aligned}
& u_{1}=(0,0,1)^{T}, \\
& u_{2}=\left(\sqrt{1-\alpha^{2}}, 0, \alpha\right)^{T}, \\
& u_{3}=\left(x_{3}, y_{3}, \alpha\right)^{T}, \\
& u_{4}=\left(x_{4}, y_{4}, \alpha\right)^{T}, \\
& u_{5}=\left(x_{5}, y_{5}, \alpha\right)^{T} .
\end{aligned}
$$

We have two cases.

Case 1. $\left|\left\langle u_{3}, u_{4}\right\rangle\right|=\alpha$.

Then the subset $\tilde{U}=\left\{u_{1}, u_{2}, u_{3}, u_{4}\right\}$ is equiangular, hence Theorem 5 implies $\alpha=1 / 3$ or $1 / \sqrt{5}$. However, just as in Lemma 5, $\alpha=1 / 3$ implies that

$$
\frac{1}{3}=\mathcal{M}_{\infty}(U) \leq \frac{1}{\sqrt{6}},
$$

and so $\alpha=1 / \sqrt{5}$.

Case 2. $\left|\left\langle u_{3}, u_{4}\right\rangle\right|<\alpha$. 
Then, since each vertex must be of degree 3 , we have that $\left|\left\langle u_{3}, u_{5}\right\rangle\right|$ and $\left|\left\langle u_{4}, u_{5}\right\rangle\right|$ each equals $\alpha$. Thus, if we remove the absolute values, we have the following equations:

$$
\begin{array}{ll}
\left\langle u_{2}, u_{3}\right\rangle= \pm \alpha, & \left\langle u_{2}, u_{4}\right\rangle= \pm \alpha, \\
\left\langle u_{3}, u_{5}\right\rangle= \pm \alpha, & \left\langle u_{4}, u_{5}\right\rangle= \pm \alpha .
\end{array}
$$

This gives $2^{4}=16$ possible cases. Of these 16 cases, 7 lead to contradictions and the remaining 9 fall into 5 types; but each implies that $u_{3}, u_{4}, u_{5}$ are three of the four points

$$
\begin{gathered}
\left(\alpha \sqrt{\frac{1-\alpha}{1+\alpha}}, \pm \sqrt{\frac{(1+2 \alpha)(1-\alpha)}{1+\alpha}}, \alpha\right)^{T}, \\
\left(-\alpha \sqrt{\frac{1+\alpha}{1-\alpha}}, \pm \sqrt{\frac{(1-2 \alpha)(1+\alpha)}{1-\alpha}}, \alpha\right)^{T},
\end{gathered}
$$

which are the positive endpoints on the remaining 4 diagonals of an icosahedron. Hence, in each case, $\alpha=1 / \sqrt{5}$.

The $(5,3)$-Grassmannian frame is the first example of a nonoptimal Grassmannian frame since $1 / \sqrt{5}>1 / \sqrt{6}$. Hence, by Theorem 3 , the $(5,3)$-Grassmannian frame is the first three-dimensional example of a Grassmannian frame which is not tight.

\section{7. (6, 3)-GRASSMANNIAN FRAMES}

The $(6,3)$-Grassmannian bound can be calculated as a consequence of Theorem 3 .

Theorem 8. If $U=\left\{u_{1}, \ldots, u_{6}\right\} \subset S^{2}$ is $(6,3)$-Grassmannian, then

$$
\mathcal{M}_{\infty}(U)=\frac{1}{\sqrt{5}} .
$$

Proof. Set $\alpha=1 / \sqrt{5}$, and consider the set $W$ with vertices

$$
\begin{aligned}
& w_{1}=(0,0,1)^{T}, \\
& w_{2}=\left(\sqrt{1-\alpha^{2}}, 0, \alpha\right)^{T}, \\
& w_{3}=\left(\alpha \sqrt{\frac{1-\alpha}{1+\alpha}}, \sqrt{\frac{(1+2 \alpha)(1-\alpha)}{1+\alpha}}, \alpha\right)^{T}, \\
& w_{4}=\left(\alpha \sqrt{\frac{1-\alpha}{1+\alpha}},-\sqrt{\frac{(1+2 \alpha)(1-\alpha)}{1+\alpha}}, \alpha\right)^{T}, \\
& w_{5}=\left(-\alpha \sqrt{\frac{1+\alpha}{1-\alpha}}, \sqrt{\frac{(1-2 \alpha)(1+\alpha)}{1-\alpha}}, \alpha\right)^{T}, \\
& w_{6}=\left(-\alpha \sqrt{\frac{1+\alpha}{1-\alpha}},-\sqrt{\frac{(1-2 \alpha)(1+\alpha)}{1-\alpha}}, \alpha\right)^{T} .
\end{aligned}
$$

Note that $\pm W$ are the twelve vertices of an icosahedron. For $k \neq l$, we compute $\left|\left\langle w_{k}, w_{l}\right\rangle\right|=1 / \sqrt{5}$. Furthermore, by Theorem 3 , if $U$ is a 6-element subset of $S^{2}$, then

$$
\mathcal{M}_{\infty}(U) \geq \sqrt{\frac{6-3}{3(6-1)}}=\frac{1}{\sqrt{5}}=\mathcal{M}_{\infty}(W) .
$$

Thus $W$ is a $(6,3)$-Grassmannian frame.
Notice the $(6,3)$ Grassmannian arrangement is so good that when one removes a vector from it, it remains Grassmannian, and when one removes two vectors from it, it is still a local minimum of $\mathcal{M}_{\infty}$. In [4] Conway, Hardin, and Sloane have found that there are other instances of this in higher dimensions, particularly when the symmetry group of the frame has a large number of elements.

\section{APPENDICES}

\section{A. EXISTENCE OF GRASSMANNIAN FRAMES}

We show that Grassmannian frames exist. First, we define the function

$$
\begin{gathered}
f: \underbrace{S^{d-1} \times \cdots \times S^{d-1}}_{N \text { times }} \longrightarrow[0,1], \\
f\left(x_{1}, \ldots, x_{N}\right)=\mathcal{M}_{\infty}\left(\left\{x_{k}\right\}_{k=1}^{N}\right) .
\end{gathered}
$$

Next we check that $f$ is continuous on $X:=\mathbb{R}^{d} \times \cdots \times \mathbb{R}^{d}$ ( $N$ times). Consider the norm on $X$ defined by

$$
\left\|\left\{x_{k}\right\}_{k=1}^{N}\right\|_{X}=\sum_{k=1}^{N}\left\|x_{k}\right\|
$$

let $\left\{x_{k}\right\}_{k=1}^{N} \in X$ be fixed, set $R-1=\max _{k}\left\{\left\|x_{k}\right\|\right\}$, and let $\varepsilon>0$ be given. Clearly $R \geq 1$. Choose $\delta$ such that $0<\delta<(\sqrt{1+\varepsilon}-1) / R$, that is, $R^{2} \delta^{2}+2 R \delta<\varepsilon$. Then, whenever $\left\|\left\{y_{k}\right\}_{k=1}^{N}-\left\{x_{k}\right\}_{k=1}^{N}\right\|_{X}<\delta$, we have that, for every $j \in\{1, \ldots, N\}$,

$$
\begin{aligned}
\left\|y_{j}-x_{j}\right\| & \leq \sum_{k=1}^{N}\left\|y_{k}-x_{k}\right\| \\
& =\left\|\left\{y_{k}\right\}_{k=1}^{N}-\left\{x_{k}\right\}_{k=1}^{N}\right\|_{X}<\delta .
\end{aligned}
$$

Therefore, for each $j$, there is an $\alpha_{j} \in \mathbb{R}^{d}$ with $\left\|\alpha_{j}\right\|<\delta$ such that $y_{j}=x_{j}+\alpha_{j}$. Thus,

$$
\begin{aligned}
& \left|f\left(y_{1}, \ldots, y_{N}\right)-f\left(x_{1}, \ldots, x_{N}\right)\right| \\
& =\max _{k \neq l}\left\{\left|\left\langle x_{k}, x_{l}\right\rangle\right|+\left|\left\langle x_{k}, \alpha_{l}\right\rangle\right|+\left|\left\langle\alpha_{k}, x_{l}\right\rangle\right|\right. \\
& \left.\quad+\left|\left\langle\alpha_{k}, \alpha_{l}\right\rangle\right|\right\}-\max _{k \neq l}\left\{\left|\left\langle x_{k}, x_{l}\right\rangle\right|\right\} \\
& \leq \max _{k \neq l}\left\{\left|\left\langle x_{k}, x_{l}\right\rangle\right|+|| x_{k}|||| \alpha_{l}||+|| \alpha_{k}|| \mid x_{l} \|\right. \\
& \left.\quad+|| \alpha_{k}|||| \alpha_{l}||\right\}-\max _{k \neq l}\left\{\left|\left\langle x_{k}, x_{l}\right\rangle\right|\right\} \\
& <\max _{k \neq l}\left\{\left|\left\langle x_{k}, x_{l}\right\rangle\right|\right\}+2 R \delta+R \delta^{2} \\
& \quad-\max _{k \neq l}\left\{\left|\left\langle x_{k}, x_{l}\right\rangle\right|\right\} \\
& =2 R \delta+R \delta^{2}<\varepsilon .
\end{aligned}
$$

Hence, $f$ is continuous on the compact set $S^{d-1} \times \cdots \times S^{d-1}$ ( $N$ times), and so $f$ achieves its absolute maximum and absolute minimum on this set. Thus, we know that $(N, d)$ Grassmannian frames exist for any $N \geq d$. Next we must check that if $U_{d}^{N}$ solves (3), then $U_{d}^{N}$ is a unit-norm frame for $\mathbb{R}^{d}$, but this a tautology since, by compactness, $U_{d}^{N}$ is one of the frames over which we are taking the infimum. 


\section{B. PROOFS OF LEMMA 1 AND THEOREM 1}

Proof of Lemma 1. We proceed by induction. Let $P(n)$ be the statement

$$
\begin{gathered}
\operatorname{det}\left(H_{n}\right)=(1+(n-1) \beta)(1-\beta)^{n-1}, \\
\operatorname{det}\left(C_{n}\right)=\beta(1-\beta)^{n-1} .
\end{gathered}
$$

For $n=1, H_{1}=1$ and $C_{1}=\beta$, and so $\operatorname{det}\left(H_{1}\right)=1$ and $\operatorname{det}\left(C_{1}\right)=\beta$; this is $P(1)$.

Next assume $P(n)$. Using the cofactor expansion of the determinant, we first note that the $(1,1)$-cofactor of $H_{n+1}$ and $C_{n+1}$ is $\operatorname{det}\left(H_{n}\right)$. Also note that for $j=2, \ldots, n+1$, the $(1, j)$ cofactor of both $H_{n+1}$ and $C_{n+1}$ is

$$
(-1)^{1+j} \operatorname{det}\left(B_{n}^{(j)}\right)
$$

where $B_{n}^{(j)}$ can be defined recursively as

$$
\begin{gathered}
B_{n}^{(1)}=C_{n}, \\
B_{n}^{(j)}=\tilde{B}_{n}^{(j-1)} \text { for } j=2, \ldots, n+1,
\end{gathered}
$$

and where $\tilde{B}_{n}^{(j-1)}$ is $B_{n}^{(j-1)}$ with the $j$ th and $(j-1)$ th rows interchanged. Since det is multilinear, interchanging a row changes the sign of the determinant. Hence,

$$
\begin{aligned}
(-1)^{1+j} & \operatorname{det}\left(B_{n}^{(j)}\right) \\
& =-\operatorname{det}\left(C_{n}\right) \text { for } j=2, \ldots, n+1 .
\end{aligned}
$$

Using the induction hypothesis and the cofactor expansion, we compute

$$
\begin{aligned}
\operatorname{det} & \left(H_{n+1}\right) \\
= & 1 \cdot \operatorname{det}\left(H_{n}\right)+\sum_{j=2}^{n+1}\left(\beta \cdot(-1)^{1+j} \operatorname{det}\left(B_{n}^{(j)}\right)\right) \\
= & \operatorname{det}\left(H_{n}\right)-n \beta \operatorname{det}\left(C_{n}\right) \\
= & (1+(n-1) \beta)(1-\beta)^{n-1}-n \beta^{2}(1-\beta)^{n-1} \\
= & (1+n \beta)(1-\beta)(1-\beta)^{n-1}, \\
\operatorname{det} & \left(C_{n+1}\right) \\
= & \beta \operatorname{det}\left(H_{n}\right)-n \beta \operatorname{det}\left(C_{n}\right) \\
= & \beta(1+(n-1) \beta)(1-\beta)^{n-1}-n \beta^{2}(1-\beta)^{n-1} \\
= & \left(\beta-\beta^{2}\right)(1-\beta)^{n-1} ;
\end{aligned}
$$

this is $P(n+1)$, and so the result follows by induction.

Proof of Theorem 3. (a) First, we prove the inequality (27). Since the $N \times N$ Grammian matrix $G$ is symmetric, the spectral theorem applies, and so $G$ has $N$ eigenvalues $\lambda_{j}$ counted with multiplicity and ordered by size, that is, $\lambda_{1} \geq \lambda_{2} \geq$ $\cdots \geq \lambda_{N}$. Furthermore, since $\operatorname{rank}(G)=d_{0}$, only the first $d_{0}$ of these eigenvalues is nonzero. Hence,

$$
\sum_{k=1}^{d_{0}} \lambda_{k}=\operatorname{Trace} G=\sum_{k=1}^{N}\left|\left\langle x_{k}, x_{k}\right\rangle\right|=\sum_{k=1}^{N} 1=N .
$$

Now set $e_{k}=\lambda_{k}-\left(N / d_{0}\right)$. Then

$$
\sum_{k=1}^{d_{0}} e_{k}=\sum_{k=1}^{d_{0}}\left(\lambda_{k}-\frac{N}{d_{0}}\right)=N-d_{0} \frac{N}{d_{0}}=0,
$$

and so

$$
\begin{aligned}
\sum_{k=1}^{d_{0}} \lambda_{k}^{2} & =\sum_{k=1}^{d_{0}}\left(\frac{N}{d_{0}}+e_{k}\right)^{2} \\
& =\sum_{k=1}^{d_{0}} \frac{N^{2}}{d_{0}^{2}}+\frac{2 N}{d_{0}} \sum_{k=1}^{d_{0}} e_{k}+\sum_{k=1}^{d_{0}} e_{k}^{2} \\
& =\frac{N^{2}}{d_{0}}+\sum_{k=1}^{d_{0}} e_{k}^{2} \geq \frac{N^{2}}{d_{0}},
\end{aligned}
$$

with equality if and only if $e_{k}=0$ for $k=1, \ldots, d_{0}$, that is, if and only if $\lambda_{k}=N / d_{0}$ for $k=1, \ldots, d_{0}$. Next, the eigenvalues of $G^{2}$ are $\lambda_{1}^{2} \geq \lambda_{2}^{2} \geq \cdots \geq \lambda_{N}^{2}$, so that if $g_{k}$ is the $k$ th column of $G$, then by matrix multiplication we have

$$
\begin{aligned}
\frac{N^{2}}{d_{0}} & \leq \sum_{k=1}^{d_{0}} \lambda_{k}^{2}=\operatorname{Trace}\left(G^{2}\right)=\sum_{k=1}^{N} g_{k}^{T} g_{k} \\
& =\sum_{k=1}^{N} \sum_{l=1}^{N}\left|\left\langle x_{k}, x_{l}\right\rangle\right|^{2} .
\end{aligned}
$$

Since $G$ is symmetric, $\left|\left\langle x_{k}, x_{l}\right\rangle\right|=\left|\left\langle x_{l}, x_{k}\right\rangle\right|$, so that by (B.9) we compute

$$
\begin{aligned}
\frac{N^{2}}{d_{0}} \leq & \sum_{k=1}^{N} \sum_{l=1}^{N}\left|\left\langle x_{k}, x_{l}\right\rangle\right|^{2} \\
= & \sum_{k=l}\left|\left\langle x_{k}, x_{l}\right\rangle\right|^{2}+\sum_{k<l}\left|\left\langle x_{k}, x_{l}\right\rangle\right|^{2} \\
& +\sum_{k>l}\left|\left\langle x_{k}, x_{l}\right\rangle\right|^{2} \\
= & N+2 \sum_{k<l}\left|\left\langle x_{k}, x_{l}\right\rangle\right|^{2} \\
\leq & N+2 \frac{N(N-1)}{2} \max _{k \neq l}\left\{\left|\left\langle x_{k}, x_{l}\right\rangle\right|^{2}\right\} .
\end{aligned}
$$

Therefore, solving for the max in (B.10), we have

$$
\frac{N-d_{0}}{d_{0}(N-1)} \leq \mathcal{M}_{\infty}\left(X_{d}^{N}\right)^{2}
$$

For future reference, we note that $d \geq d_{0}$ implies $(N-$ $d) /(d(N-1)) \leq\left(N-d_{0}\right) /\left(d_{0}(N-1)\right)$. Hence, $(27)$ remains true when we replace $d$ with $d_{0} \leq d$.

(b) Next we prove that equality holds in (27) if and only if $X_{d}^{N}$ is equiangular and is a tight frame for its span.

$(\Longrightarrow)$. Suppose $\mathcal{M}_{\infty}\left(X_{d}^{N}\right)=\sqrt{\left(N-d_{0}\right) /\left(d_{0}(N-1)\right)}$. Then (B.10) becomes

$$
\sum_{k=1}^{N} \sum_{l=1}^{N}\left|\left\langle x_{k}, x_{l}\right\rangle\right|^{2}=\frac{N^{2}}{d_{0}}
$$


which implies from (B.9) that

$$
\sum_{k=1}^{d_{0}} \lambda_{k}^{2}=\frac{N^{2}}{d_{0}}
$$

and, as we saw above, equality in this sum implies that $\lambda_{k}=N / d_{0}$ for $k=1, \ldots, d_{0}$. The frame bounds for $X_{d}^{N}$ are the largest and smallest nonzero eigenvalues, and hence $A=N / d_{0}=B$ and so $X_{d}^{N}$ is a tight frame for its span.

To see that $X_{d}^{N}$ is also equiangular, we notice that (B.10) gives

$$
N+2 \sum_{k<l}\left|\left\langle x_{k}, x_{l}\right\rangle\right|^{2}=\frac{N^{2}}{d_{0}}
$$

and hence

$$
\sum_{k<l}\left|\left\langle x_{k}, x_{l}\right\rangle\right|^{2}=\frac{N\left(N-d_{0}\right)}{2 d_{0}}
$$

Our assumption $\max _{k \neq l}\left|\left\langle x_{k}, x_{l}\right\rangle\right|^{2}=N-d_{0} / d_{0}(N-1)$ implies that, for any $k \neq l$,

$$
\left|\left\langle x_{k}, x_{l}\right\rangle\right|^{2}=\frac{N-d_{0}}{d_{0}(N-1)}-\varepsilon_{k, l},
$$

where $\varepsilon_{k, l} \geq 0$. Thus (B.15) is

$$
\begin{aligned}
\frac{N\left(N-d_{0}\right)}{2 d_{0}} & =\sum_{k<l}\left(\frac{N-d_{0}}{d_{0}(N-1)}-\varepsilon_{k, l}\right) \\
& =\left(\frac{N(N-1)}{2}\right)\left(\frac{N-d_{0}}{d_{0}(N-1)}\right)-\sum_{k<l} \varepsilon_{k, l} \\
& =\frac{N\left(N-d_{0}\right)}{2 d_{0}}-\sum_{k<l} \varepsilon_{k, l} .
\end{aligned}
$$

Therefore, $\sum_{k<l} \varepsilon_{k, l}=0$, and since the $\varepsilon_{k, l}$ are nonnegative, we can assert that $\varepsilon_{k, l}=0$ for $k<l$. Also, since $G$ is symmetric, $\varepsilon_{k, l}=0$ for all $k \neq l$, and hence $X_{d}^{N}$ is equiangular with $\left|\left\langle x_{k}, x_{l}\right\rangle\right|^{2}=\left(N-d_{0}\right) /\left(d_{0}(N-1)\right)$.

$(\Longleftarrow)$. Now assume $X_{d}^{N}$ is equiangular and is a tight frame for its span with frame bound $A=B=N / d_{0}$. Then there is an $\alpha \in[0,1]$, such that $\left|\left\langle x_{k}, x_{l}\right\rangle\right|^{2}=\alpha$ for $k \neq l$. Now since $X_{d}^{N}$ is tight, $\lambda_{k}=N / d_{0}$ for $k=1, \ldots, d_{0}$, and zero otherwise. Hence, (B.9) and (B.10) imply

$$
\frac{N^{2}}{d_{0}}=\sum_{k=1}^{d_{0}} \lambda_{k}^{2}=\sum_{k=1}^{N} \sum_{l=1}^{N}\left|\left\langle x_{k}, x_{l}\right\rangle\right|^{2}=N+N(N-1) \alpha .
$$

Thus, solving for $\alpha$, we see that equality holds in (27).

(c) Finally, to prove $N>d(d+1) / 2$ implies $X_{d}^{N}$ is not equiangular, we prove the contrapositive using Lemma 1 and the following argument (cf. [21]). Assume $X_{d}^{N}$ is equiangular. Let $P_{k}: \mathbb{R}^{d} \rightarrow \mathbb{R}^{d}$ be the projection of $x$ onto the line spanned by $x_{k}$, that is, $P_{k} x=\left\langle x, x_{k}\right\rangle x_{k}$. Let $V$ be the vector space of symmetric linear mappings $\mathbb{R}^{d} \rightarrow \mathbb{R}^{d}$. Then $\operatorname{dim}(V)=d(d+1) / 2$, and the map $\langle\cdot, \cdot\rangle: V \times V \rightarrow \mathbb{R}$ given by $\langle C, D\rangle=\operatorname{Trace}(C D)$ is an inner product on $V$. Since $X_{d}^{N}$ is equiangular, there is an $\alpha \in[0,1]$ such that $\left\langle x_{k}, x_{l}\right\rangle= \pm \alpha$ for $k \neq l$. Furthermore, $\alpha=1$ implies $N=2$, since the elements of $X_{d}^{N}$ are assumed to be distinct and of unit norm. Thus, for $d \geq 2$, we have $N=2<3 \leq d(d+1) / 2$. Therefore, we may assume $\alpha \in[0,1)$. Now,

$$
\left\langle P_{k}, P_{l}\right\rangle=\left\langle x_{k}, x_{l}\right\rangle^{2}= \begin{cases}1, & \text { if } k=l \\ \alpha^{2}, & \text { if } k \neq l\end{cases}
$$

Hence, the Grammian of the set $\left\{P_{1}, \ldots, P_{N}\right\} \subset V$ is

$$
[G]_{k, l}=\left[\left\langle P_{k}, P_{l}\right\rangle\right]_{k, l}= \begin{cases}1, & \text { if } k=l, \\ \alpha^{2}, & \text { if } k \neq l,\end{cases}
$$

where $[G]_{k, l}$ is the $(k, l)$ th entry of the matrix $G$. Thus, Lemma 1 applies with $G=H_{N}$ and $\beta=\alpha^{2}$. Consequently, if $\alpha \in[0,1)$, then

$$
\operatorname{det} G=\left(1+(N-1) \alpha^{2}\right)\left(1-\alpha^{2}\right)^{N-1} \neq 0 .
$$

Therefore, $G$ is invertible and has full rank. Finally, since $\operatorname{rank}(G)=\operatorname{rank}(S)=N$, we have that

$$
\begin{aligned}
N & =\operatorname{rank}(G)=\operatorname{dim}\left(\operatorname{span}\left\{P_{1}, \ldots, P_{N}\right\}\right) \\
& \leq \operatorname{dim}(V)=\frac{d(d+1)}{2} .
\end{aligned}
$$

We have proved that if $X_{d}^{N}$ is equiangular, then $N \leq d(d+$ $1) / 2$; and so, by contraposition, we have proven the result.

\section{PROOF OF PROPOSITION 3}

Proof of Proposition 3. Let $v \in Q \backslash C$. Then there is a $\lambda \in$ $(0,1)$, and there are $x_{1}, x_{2} \in Q$ with $x_{1} \neq x_{2}$ such that $v=$ $\lambda x_{1}+(1-\lambda) x_{2}$. Consider the function $f: \mathbb{R} \rightarrow \mathbb{R}$ defined by

$$
f(\lambda)=\left\|\lambda x_{1}+(1-\lambda) x_{2}\right\|
$$

We first verify that $f$ is uniformily continuous on $\mathbb{R}$. Let $\varepsilon>0$ be given, and choose $\delta<\varepsilon /\left\|x_{1}-x_{2}\right\|$. Then, for all $\lambda_{0}$ for which $\left|\lambda-\lambda_{0}\right|<\delta$, we have

$$
\begin{aligned}
\mid f & (\lambda)-f\left(\lambda_{0}\right) \mid \\
& =\left\|\lambda x_{1}+(1-\lambda) x_{2}\right\|-\left\|\lambda_{0} x_{1}+\left(1-\lambda_{0}\right) x_{2}\right\| \mid \\
& \leq\left\|\lambda x_{1}+(1-\lambda) x_{2}-\lambda_{0} x_{1}-\left(1-\lambda_{0}\right) x_{2}\right\| \\
& =\left\|\left(\lambda-\lambda_{0}\right)\left(x_{1}-x_{2}\right)\right\| \\
& =\left|\lambda-\lambda_{0}\right|\left\|x_{1}-x_{2}\right\| \\
& <\delta\left\|x_{1}-x_{2}\right\|<\varepsilon .
\end{aligned}
$$

Now set $g(\lambda)=f(\lambda)^{2}$. Then $g(\lambda)$ is also continuous on $\mathbb{R}$ and it is the parabola,

$$
\begin{aligned}
g(\lambda) & =\left\|\lambda x_{1}+(1-\lambda) x_{2}\right\|^{2} \\
& =\lambda^{2}\left\|x_{1}\right\|^{2}+2\left(\lambda-\lambda^{2}\right)\left\langle x_{1}, x_{2}\right\rangle-2(1-\lambda)\left\|x_{2}\right\|^{2} .
\end{aligned}
$$


We compute

$$
g^{\prime}(\lambda)=2 \lambda\left\|x_{1}-x_{2}\right\|^{2}+2\left\langle x_{1}-x_{2}, x_{2}\right\rangle,
$$

so that $g^{\prime}(\lambda)=0$ at

$$
\lambda_{*}=-\frac{\left\langle x_{1}-x_{2}, x_{2}\right\rangle}{\left\|x_{1}-x_{2}\right\|^{2}} .
$$

Furthermore, for all $\lambda \in \mathbb{R}$,

$$
g^{\prime \prime}(\lambda)=2\left\|x_{1}-x_{2}\right\|^{2}>0 .
$$

Hence, $g$ attains a minimum at $\lambda_{*}$, and for all $\lambda \neq \lambda_{*}$, we have $g(\lambda)>g\left(\lambda_{*}\right)$. Now if we restrict $g$ to $[0,1]$, then $g$ achieves its maximum and minimum on $[0,1]$. Thus, if $\lambda_{*} \in[0,1]$, then by (C.6) and the fact that $g$ describes a parabola, we have

$$
\begin{gathered}
\min _{\lambda \in[0,1]} g(\lambda)=g\left(\lambda_{*}\right), \\
\max _{\lambda \in[0,1]} g(\lambda)=\max \{g(0), g(1)\},
\end{gathered}
$$

and if $\lambda_{*} \notin[0,1]$, then

$$
\begin{aligned}
& \min _{\lambda \in[0,1]} g(\lambda)=\min \{g(0), g(1)\}, \\
& \max _{\lambda \in[0,1]} g(\lambda)=\max \{g(0), g(1)\} .
\end{aligned}
$$

In either case, the maximum of $g$ occurs at one of the end points. Furthermore, at interior points, $g$ is strictly less than the maximum value.

Now since $\|v\|^{2}=g\left(\lambda_{0}\right)$ for some $\lambda_{0} \in(0,1),($ C.6) implies

$$
\begin{aligned}
\|v\|^{2} & =g\left(\lambda_{0}\right) \\
& <\max _{\lambda \in[0,1]} g(\lambda)=\max \{g(0), g(1)\} \\
& =\max \left\{\left\|x_{1}\right\|^{2},\left\|x_{2}\right\|^{2}\right\} .
\end{aligned}
$$

Thus, we have shown that

$$
v \in Q \backslash C \Longrightarrow \exists x \in Q \text { such that }\|v\|<\|x\|,
$$

and hence

$$
v \in Q \backslash C \Longrightarrow\|v\|<\sup \{\|x\|: x \in Q\} .
$$

$Q$ is a bounded closed set so that, by continuity of $\|\cdot\|$, the supremum in (C.11) is achieved on $Q$, whereas (C.11) also shows that this supremum is not achieved on $Q \backslash C$. Thus,

$$
\sup \{\|x\|: x \in Q\}=\sup \{\|x\|: x \in C\}=\|c\| .
$$

Therefore, for any $v \in Q \backslash C$, we have $\|v\|<\sup \{\|x\|: x \in$ $Q\}=\|c\|$.

\section{ACKNOWLEDGMENTS}

Both authors gratefully acknowledge the support from NSF DMS Grant 0139759 and ONR Grant N000140210398. We also greatly appreciate the perspicacious and constructive observations by the three referees.

\section{REFERENCES}

[1] O. Christensen, An Introduction to Frames and Riesz Bases, Birkhäuser, Boston, Mass, USA, 2002.

[2] T. Strohmer and R. W. Heath Jr., "Grassmannian frames with applications to coding and communication," Applied and Computational Harmonic Analysis, vol. 14, no. 3, pp. 257-275, 2003.

[3] J. J. Benedetto and M. Fickus, "Finite normalized tight frames," Advances in Computational Mathematics, vol. 18, no. 2-4, pp. 357-385, 2003.

[4] J. H. Conway, R. H. Hardin, and N. J. A. Sloane, "Packing lines, planes, etc.: packings in Grassmannian spaces," Experimental Mathematics, vol. 5, no. 2, pp. 139-159, 1996.

[5] Y. C. Eldar and H. Bölcskei, "Geometrically uniform frames," IEEE Transactions on Informormation Theory, vol. 49, no. 4, pp. 993-1006, 2003.

[6] J. A. Tropp, I. S. Dhillon, R. W. Heath Jr., and T. Strohmer, "Designing structured tight frames via an alternating projection method," IEEE Transactions on Informormation Theory, vol. 51, no. 1, pp. 188-209, 2005.

[7] V. K. Goyal, J. Kovačević, and J. A. Kelner, "Quantized frame expansions with erasures," Applied and Computational Harmonic Analysis, vol. 10, no. 3, pp. 203-233, 2001.

[8] P. G. Casazza and J. Kovačević, "Equal-norm tight frames with erasures," Advances in Computational Mathematics, vol. 18, no. 2-4, pp. 387-430, 2003.

[9] R. Holmes and V. Paulsen, "Optimal frames for erasures," Linear Algebra and its Applications, vol. 377, no. 1, pp. 31-51, 2004.

[10] L. Fejes Tóth, "Distribution of points in the elliptic plane," Acta Mathematica Academiae Scientiarum Hungaricae, vol. 16, pp. 437-440, 1965.

[11] D. C. Lay, Linear Algebra and Its Applications, Addison-Wesley, Boston, Mass, USA, 2003.

[12] R. J. Duffin and A. C. Schaeffer, "A class of nonharmonic Fourier series," Transactions of the American Mathematical Society, vol. 72, no. 2, pp. 341-366, 1952.

[13] J. J. Benedetto and M. W. Frazier, Eds., Wavelets: Mathematics and Applications, CRC Press, Boca Raton, Fla, USA, 1994.

[14] I. Daubechies, Ten Lectures on Wavelets, SIAM, Philadelphia, Pa, USA, 1992.

[15] M. Rosenfeld, "In praise of the Gram matrix," in The Mathematics of Paul Erdo's, II, vol. 14 of Algorithms and Combinatorics, pp. 318-323, Springer, Berlin, Germany, 1997.

[16] L. R. Welch, "Lower bounds on the maximum crosscorrelation of signals," IEEE Transactions on Informormation Theory, vol. 20, no. 3, pp. 397-399, 1974.

[17] M. J. Panik, Fundamentals of Convex Analysis: Duality, Separation, Representation, and Resolution, Kluwer Academic, Dordrecht, the Netherlands, 1993.

[18] S. R. Lay, Convex Sets and Their Applications, John Wiley \& Sons, New York, NY, USA, 1982.

[19] R. Webster, Convexity, Oxford University Press, New York, NY, USA, 1994.

[20] M. Krein and D. Milman, "On extreme points of regular convex sets," Studia Mathematica, vol. 9, pp. 133-138, 1940.

[21] P. W. H. Lemmens and J. J. Seidel, "Equiangular lines," Journal of Algebra, vol. 24, no. 3, pp. 494-512, 1973. 
John J. Benedetto received the B.A. degree from Boston College in 1960, the M.A. degree from Harvard University in 1962, and the Ph.D. degree from the University of Toronto in 1964. All degrees are in mathematics. He is a Professor of mathematics at University of Maryland, the Director of the Norbert Wiener Center in the Department of Mathematics, and a Distinguished Scholar-Teacher of the University. He has

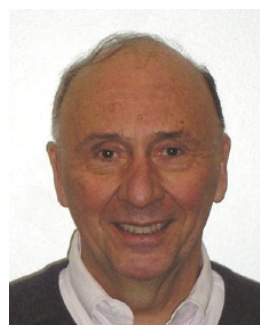
published 150 research papers in harmonic analysis and its applications, directed 40 doctoral dissertations, and consulted for The MITRE Corporation from 1987 to 1996. He is the Founding Editorin-Chief and Executive Editor of the Journal of Fourier Analysis and Applications and the Series Editor of Birkhauser's Applied and Numerical Harmonic Analysis book series.

Joseph D. Kolesar recieved the B.S. degree in mathematics from Duquesne University in 1997 and the M.S. degree in mathematics from the University of Vermont in 1999. In 2004 he received the Ph.D. degree in mathematics from the University of Maryland at College Park. He is currently working with the US government as an Applied Research Mathematician.

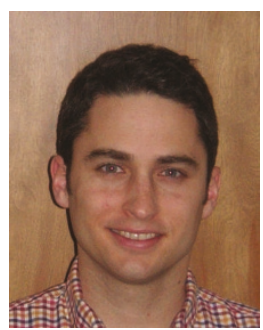

